\title{
Pharmacokinetics and Exposure-Response of Vosoritide in Children with Achondroplasia
}

\author{
Ming Liang Chan ${ }^{1}(1) \cdot$ Yulan $\mathrm{Qi}^{1} \cdot$ Kevin Larimore $^{1} \cdot$ Anu Cherukuri $^{1} \cdot$ Lori Seid $^{1} \cdot$ Kala Jayaram $^{1} \cdot$ George Jeha $^{1}$. \\ Elena Fisheleva ${ }^{2} \cdot$ Jonathan Day $^{2}$ - Alice Huntsman-Labed ${ }^{2} \cdot$ Ravi Savarirayan $^{3} \cdot$ Melita Irving $^{4}$ - Carlos A. Bacino ${ }^{5}$. \\ Julie Hoover-Fong ${ }^{6} \cdot$ Keiichi Ozono $^{7} \cdot$ Klaus Mohnike $^{8} \cdot$ William R. Wilcox $^{9} \cdot$ William A. Horton $^{10}$. Joshua Henshaw ${ }^{1}$
}

Accepted: 11 July 2021 / Published online: 25 August 2021

(c) The Author(s) 2021

\begin{abstract}
Background and Objective Vosoritide, an analog of C-type natriuretic peptide, has been developed for the treatment of children with achondroplasia. The pharmacokinetics of vosoritide and relationships between plasma exposure and efficacy, biomarkers, and safety endpoints were evaluated in a phase II, open-label, dose-escalation study $(N=35$ patients aged 5-14 years who received daily subcutaneous injections for 24 months) and a phase III, double-blind, placebo-controlled study ( $N$ $=60$ patients aged 5-18 years randomized to receive daily subcutaneous injections for 52 weeks).

Methods Pharmacokinetic parameters for both studies were obtained from non-compartmental analysis. Potential correlations between vosoritide exposure and changes in annualized growth velocity, collagen type X marker (CXM; a biomarker of endochondral ossification), cyclic guanosine monophosphate (cGMP; a biomarker of pharmacological activity), heart rate, and systolic and diastolic blood pressures were then evaluated.

Results The exposure-response relationships for changes in both annualized growth velocity and the CXM biomarker saturated at $15 \mu \mathrm{g} / \mathrm{kg}$, while systemic pharmacological activity, as measured by urinary cGMP, was near maximal or saturated at exposures obtained at the highest dose studied (i.e. $30 \mu \mathrm{g} / \mathrm{kg}$ ). This suggested that the additional bioactivity was likely in tissues not related to endochondral bone formation. In the phase III study, following subcutaneous administration at the recommended dose of $15 \mu \mathrm{g} / \mathrm{kg}$ to patients with achondroplasia aged 5-18 years, vosoritide was rapidly absorbed with a median time to maximal plasma concentration $\left(C_{\max }\right)$ of 15 minutes, and cleared with a mean half-life of 27.9 minutes after 52 weeks of treatment. Vosoritide exposure ( $C_{\max }$ and area under the concentration-time curve [AUC]) was consistent across visits. No evidence of accumulation with once-daily dosing was observed. Total anti-vosoritide antibody (TAb) responses were detected in the serum of 25 of 60 (42\%) treated patients in the phase III study, with no apparent impact of TAb development noted on annualized growth velocity or vosoritide exposure. Across the exposure range obtained with $15 \mu \mathrm{g} / \mathrm{kg}$ in the phase III study, no meaningful correlations between vosoritide plasma exposure and changes in annualized growth velocity or CXM, or changes from predose heart rate, and systolic or diastolic blood pressures were observed.

Conclusions The results support the recommended dose of vosoritide $15 \mu \mathrm{g} / \mathrm{kg}$ for once-daily subcutaneous administration in patients with achondroplasia aged $\geq 5$ years whose epiphyses are not closed.

Clinical Trials Registration NCT02055157, NCT03197766, and NCT01603095.
\end{abstract}

\section{Introduction}

Achondroplasia is the most common form of disproportionate short stature and is characterized by rhizomelic shortening of the limbs and macrocephaly [1-3]. Achondroplasia is an inherited autosomal dominant chondrodysplasia caused

Ming Liang Chan

daniel.qspharma@gmail.com

Extended author information available on the last page of the article by a single nucleotide gain-of-function pathogenic variant in the fibroblast growth factor receptor 3 (FGFR3) gene. The resultant protein product constitutively activates the mitogen-activated protein kinase (MAPK)/extracellular signal-regulated kinase (ERK) inhibitory signaling pathway in chondrocytes, inhibiting endochondral ossification [4-6]. Achondroplasia is associated with a condition-specific developmental milestone profile, functional limitations impacting quality of life, causing chronic pain and leading to psychosocial challenges [7-10]. Medical complications 


\section{Key Points}

The pharmacokinetics of vosoritide and relationships between vosoritide plasma exposure and efficacy, biomarkers, and safety endpoints were evaluated in a phase II and a phase III study.

The exposure-response relationships for annualized growth velocity and an endochondral ossification biomarker saturated at exposures obtained at $15 \mu \mathrm{g} /$ $\mathrm{kg}$, while pharmacological activity was near maximal at exposures obtained at $30 \mu \mathrm{g} / \mathrm{kg}$. No differences were observed in the safety profile of vosoritide between the two doses.

The results from these analyses support the recommended dose of $15 \mu \mathrm{g} / \mathrm{kg}$ vosoritide for once-daily subcutaneous administration in patients with achondroplasia aged $\geq 5$ years whose growth plates are not closed.

include foramen magnum stenosis, cervicomedullary compression, hydrocephalus, hypotonia, back and leg pain, and hearing loss and speech delay related to recurrent otitis media and chronic middle ear fluid. There are no approved pharmacological therapies for achondroplasia except for growth hormone, which is available for this indication in Japan [11]; however, while it may help accelerate growth in the first 2 years of treatment, its effect on disproportionality and long-term complications are unknown [12-14]. Limb lengthening procedures are available to patients but they are controversial and are associated with a high risk of complications [15].

Natriuretic peptides comprise a family of three structurally related peptides: atrial natriuretic peptide, brain natriuretic peptide, and C-type natriuretic peptide (CNP). $\mathrm{CNP}$, encoded by the Nppc gene, and its receptor, natriuretic peptide receptor 2 (NPR-B), are potent stimulators of endochondral ossification [16]. Distinct domains of expression of FGFR3 and NPR-B in the growth plate allow these two antagonistic signaling pathways to maintain a finely balanced rate of chondrocyte hypertrophy [17]. Reduced or absent $N p p c$ or $N p r 2$ gene expression in mice results in severe dwarfism due to impaired endochondral ossification $[18,19]$. Conversely, overexpression of the Nppc gene in mice and human disease models with NPPC overexpression are characterized by sustained skeletal overgrowth [20-23]. Endogenous CNP overproduction and continuous intravenous infusion of exogenous CNP rescues the impaired bone growth observed in mice with achondroplasia and increases long bone growth in wild-type monkeys through inhibition of the FGFR3-mediated MAPK signaling pathway, supporting the potential for CNP-targeted therapies in achondroplasia [19, 24-26].

Vosoritide (molecular weight $4.1 \mathrm{kD}$ ) is an analog of CNP that was designed to mimic CNP receptor binding and pharmacological activity, with structural modification to confer resistance to neutral endopeptidase (NEP) digestion in order to have an extended half-life in comparison with CNP. Like CNP, vosoritide binding to NPR-B stimulates intracellular cyclic guanosine monophosphate (cGMP) production and inhibits FGFR3 downstream signaling cascade. This in turn counteracts the effect of constitutive FGFR3 activation on chondrocyte function and promotes endochondral bone growth by stimulating chondrocyte proliferation and differentiation [27-29].

Type $\mathrm{X}$ collagen is a homotrimeric protein consisting of the $\mathrm{C}$-terminal $\mathrm{NC} 1$ and $\mathrm{N}$-terminal NC2 domains connected by a collagenous triple helix [30]. It is produced by hypertrophic chondrocytes and promotes endochondral ossification by gathering matrix components into the hypertrophic zone of the cartilage and modulating cartilage and bone mineralization [31]. As endochondral ossification occurs, the $\mathrm{NC} 1$ domain of type $\mathrm{X}$ collagen is released into the blood and this CXM biomarker was found to correlate with skeletal growth velocity in humans and mice $[30,32]$. As such, in the vosoritide development program, CXM was measured in serum as an exploratory biomarker to investigate the effect of vosoritide on growth plate activity. Both cGMP and CXM biomarkers were measured to assess pharmacodynamic (PD) responses following vosoritide treatment in patients with achondroplasia.

The safety and efficacy of vosoritide have been investigated in a phase II, open-label, dose-escalation and extension study [33] and a double-blind, placebo-controlled, phase III study [34]. In the phase II study, long-term administration of vosoritide at daily doses of both 15 and $30 \mu \mathrm{g} /$ $\mathrm{kg}$ for up to 52 months was generally well-tolerated with no change in safety profile over time. No differences were observed in the safety profile of vosoritide between the two doses. No meaningful differences were observed in the pattern or timing of heart rate, and systolic or diastolic blood pressure changes between patients receiving 15 and $30 \mu \mathrm{g} /$ $\mathrm{kg}$ in clinical studies. The phase II study demonstrated a sustained mean increase in annualized growth velocity (AGV) of $1.55 \mathrm{~cm} /$ year above baseline up to 42 months in children with achondroplasia administered a daily dose of $15 \mu \mathrm{g} / \mathrm{kg}$. The phase III study showed the adjusted mean difference in AGV between patients in the vosoritide and placebo groups was $1.57 \mathrm{~cm} /$ year in favor of vosoritide $(95 \%$ confidence interval [CI] 1.22-1.93; two-sided $p<0.0001$ ).

A more detailed summary of the pharmacokinetic (PK) data from the phase II study presented previously [33] is 
shown in Table 1. After a single subcutaneous dose of vosoritide at $2.5,7.5,15$ or $30 \mu \mathrm{g} / \mathrm{kg}$, the median time to maximal plasma concentration $\left(T_{\max }\right)$ ranged from 5 to $16 \mathrm{~min}$, and the mean of the maximum observed plasma concentration $\left(C_{\max }\right)$ and area under the concentration-time curve from time zero to the time of the last measurable concentration $\left(\mathrm{AUC}_{0-\mathrm{t}}\right)$ ranged from 549 to $16,800 \mathrm{pg} / \mathrm{mL}$ and 1370 to $689,000 \mathrm{pg}-\mathrm{min} / \mathrm{mL}$, respectively. PK data from the phase II and III studies were used to analyze the relationships between plasma exposure and efficacy, biomarkers, and safety endpoints.

\section{Methods}

\subsection{Clinical Study Design}

In the phase II dose-finding study (ClinicalTrials.gov NCT02055157; date of registration, 5 February 2014), 35 patients with achondroplasia aged 5-14 years were administered daily subcutaneous injections of $2.5,7.5,15$ or $30 \mu \mathrm{g} /$ $\mathrm{kg}$ vosoritide for 6 months, followed by an optional openlabel extension phase of 18 months where patients who received 2.5 or $7.5 \mu \mathrm{g} / \mathrm{kg}$ were subsequently administered $15 \mu \mathrm{g} / \mathrm{kg}$ vosoritide, while those patients who received 15 or $30 \mu \mathrm{g} / \mathrm{kg}$ continued to receive their initial doses. In the phase III, randomized, double-blind, placebo-controlled study (ClinicalTrials.gov NCT03197766; date of registration, 23 June 2017), a total of 121 patients aged 5-18 years were enrolled. 60 patients were randomized to receive daily subcutaneous injections of $15 \mu \mathrm{g} / \mathrm{kg}$ vosoritide for 52 weeks.

\subsection{Sample Collections and Bioanalytical Methods}

Vosoritide concentrations were measured in plasma samples for PK analyses in the phases II and III studies. During the initial 6 months of the phase II study, full PK sampling was performed at days 1, 10, 29, 85 and 183, during which plasma samples were collected predose and at $5( \pm 2 \mathrm{~min})$, 15 ( $\pm 2 \mathrm{~min}), 30$ ( $\pm 5 \mathrm{~min}), 60$ ( $\pm 5 \mathrm{~min}), 90$ ( $\pm 5 \mathrm{~min}), 120$ $( \pm 5 \mathrm{~min})$ and $180( \pm 5 \mathrm{~min}) \mathrm{min}$ postdose. For patients whose doses were not changed in the extension phase, full PK sampling was performed at months 12 and 24, while sparse PK sampling was performed at months $8,10,15,18$ and 21, during which plasma samples were collected predose and at $15( \pm 2 \mathrm{~min}), 30( \pm 5 \mathrm{~min})$, and $60( \pm 5 \mathrm{~min})$ $\mathrm{min}$ postdose. For patients whose doses were adjusted from 2.5 or $7.5 \mu \mathrm{g} / \mathrm{kg}$ to $15 \mu \mathrm{g} / \mathrm{kg}$ in the extension phase, full PK sampling was performed at the dose adjustment visit and sparse PK sampling was performed 4 weeks after the adjustment. Thereafter, PK sampling followed the same schedule as those patients who were not dose-escalated. In the phase III study, full PK sampling was performed at day 1 , week 26 , and week 52, during which plasma samples were collected predose and at $5( \pm 2 \mathrm{~min}), 15( \pm 2 \mathrm{~min}), 30( \pm 5$ $\mathrm{min}), 45$ ( $\pm 5 \mathrm{~min}), 60$ ( $\pm 5 \mathrm{~min}), 90( \pm 5 \mathrm{~min})$ and $120( \pm$ $5 \mathrm{~min}$ ) min postdose. Sparse PK sampling was performed at week 13 and week 39, during which plasma samples were collected predose and at $15( \pm 2 \mathrm{~min}), 30( \pm 5 \mathrm{~min})$, and 60

Table 1 Summary of vosoritide PK parameters after a single dose in the phase II study

\begin{tabular}{|c|c|c|c|c|c|c|c|c|c|c|}
\hline Cohort & Dose $(\mu \mathrm{g} / \mathrm{kg})$ & Visit & Statistic & $T_{\max }(\min )$ & $C_{\max }(\mathrm{pg} / \mathrm{mL})$ & $\begin{array}{l}\mathrm{AUC}_{0-\mathrm{t}}(\mathrm{pg}-\mathrm{min} / \\
\mathrm{mL})\end{array}$ & $\begin{array}{l}\mathrm{AUC}_{0-\infty} \\
\text { (pg-min/ } \\
\mathrm{mL})\end{array}$ & $\begin{array}{l}\mathrm{CL} / \mathrm{F} \\
(\mathrm{mL} / \mathrm{min} / \\
\mathrm{kg})\end{array}$ & $V_{\mathrm{z}} / F(\mathrm{~mL} / \mathrm{kg})$ & $t_{1 / 2}(\min )$ \\
\hline \multirow[t]{3}{*}{$\mathrm{C} 1$} & 2.5 & Day 1 & Mean & 5.00 & 549 & 1370 & NA & NA & NA & NA \\
\hline & & & SD & - & - & - & NA & NA & NA & NA \\
\hline & & & CV\% & - & - & - & NA & NA & NA & NA \\
\hline \multirow[t]{3}{*}{$\mathrm{C} 2$} & 7.5 & Day 1 & Mean & 13.7 & 1360 & 32,200 & NA & NA & NA & NA \\
\hline & & & SD & 6.26 & 901 & 28,100 & NA & NA & NA & NA \\
\hline & & & CV\% & 45.7 & 66.3 & 87.3 & NA & NA & NA & NA \\
\hline \multirow[t]{3}{*}{ C3 } & 15 & Day 1 & Mean & 15.8 & 4750 & 175,000 & 293,000 & 83.7 & 2560 & 24.4 \\
\hline & & & SD & 5.98 & 1990 & 113,000 & 258,000 & 73.8 & 1730 & 7.17 \\
\hline & & & CV\% & 37.8 & 41.8 & 64.6 & 88.1 & 88.1 & 67.5 & 29.4 \\
\hline \multirow[t]{3}{*}{$\mathrm{C} 4$} & 30 & Day 1 & Mean & 14.4 & 16,800 & 689,000 & 779,000 & 49.2 & 1830 & 27.0 \\
\hline & & & SD & 7.26 & 11,500 & 383,000 & 393,000 & 28.4 & 1020 & 7.72 \\
\hline & & & CV\% & 50.3 & 68.7 & 55.6 & 50.5 & 57.7 & 55.6 & 28.6 \\
\hline
\end{tabular}

All summary statistics rounded to three significant figures

$A U C_{0-\infty}$ area under the plasma concentration-time curve from time zero to infinity, $A U C_{0-t}$ area under the plasma concentration-time curve from time zero to the time of the last measurable concentration, $C L / F$ apparent clearance, $C_{\max }$ maximum observed plasma concentration, $C V$ coefficient of variation, $N A$ not available due to insufficient data points for the calculation of pharmacokinetic parameters, $S D$ standard deviation, $t_{1 / 2}$ half-life, $T_{\max }$ time to reach maximum concentration, $V_{z} / F$ apparent volume of distribution 
( $\pm 5 \mathrm{~min}$ ) min postdose. Whole blood was collected at each time point in $1 \mathrm{~mL}$ vacutainer collection tubes containing tripotassium ethylene di-amine tetra-acetic acid $\left(\mathrm{K}_{3}\right.$-EDTA), phosphodiesterase inhibitor (3-Isobutyl-1-methylxanthine; Sigma-Aldrich catalog \#I5879) and a cocktail of protease inhibitors (AEBSF, Aprotinin, Bestatin, E-64, EDTA, and Leupeptin; Sigma-Aldrich catalog \#P2714).

\subsubsection{Quantitative Measurement of Vosoritide in Human Plasma}

In the phase II study, plasma samples were analyzed for vosoritide concentrations using a validated enzyme-linked immunosorbent assay (ELISA). The assay used a murine monoclonal anti-vosoritide immunoglobulin (Ig) G capture antibody and a rabbit polyclonal anti-CNP IgG primary detection antibody. The assay utilized horseradish peroxidase-conjugated goat anti-rabbit IgG for secondary detection and tetramethyl benzidine (TMB) substrate for colorimetric development. The optical density (OD) at $450 \mathrm{~nm}$ was read for each well using a SpectraMax spectrophotometer (Molecular Devices, San Jose, CA, USA). The concentration of vosoritide in study samples was determined by interpolation of raw OD signals for each sample against a standard calibrator curve prepared in human plasma, using a fourparameter logistic curve fit. The assay lower limit of quantitation (LLOQ) was $391 \mathrm{pg} / \mathrm{mL}$ vosoritide in neat plasma.

The vosoritide PK ELISA was further optimized as an electrochemiluminesence assay (ECLA) before analysis of phase III study samples. In the PK ECLA, the capture reagent was a biotinylated mouse monoclonal anti-vosoritide $\mathrm{IgG}$, and the detection reagent was a ruthenylated rabbit polyclonal anti-vosoritide IgG. Meso Scale Discovery (MSD) read buffer was used for the generation of ECL signal, which was recorded using an MSD Quickplex instrument. The concentration of vosoritide in study samples was determined by interpolation of raw assay signals against the standard calibrator curve, using a four-parameter logistic curve fit. The assay LLOQ was $137 \mathrm{pg} / \mathrm{mL}$ vosoritide in neat plasma.

\subsubsection{Relative Quantitative Measurement of Cyclic Guanosine Monophosphate (cGMP) in Human Urine}

Cyclic guanosine monophosphate (cGMP) was measured in urine as an exploratory biomarker of overall vosoritide activity through the target receptor NPR-B. Potential sources of cGMP include the target tissue (growth plate chondrocytes) and other tissues that express NPR-B, such as vascular endothelium. Urine samples were collected for cGMP measurement before dose administration and at 1,2 and $4 \mathrm{~h}$ after dosing. In the phase II study, samples were collected on days $1,10,15,29,43,85,127$ and 183 in the initial 6-month period, and at months 12,18 and 24 of the extension phase.
In the phase III study, samples were collected on days 1,2 and 3 , and weeks 6,26 and 52 .

A relative quantitative competitive ELISA based on an assay kit from Cayman Chemical Co. (Ann Arbor, MI, USA) was developed and qualified to measure cGMP in human urine samples from the phase III study. The method was later validated before testing phase III urine samples. In the method, cGMP in diluted urine samples and a cGMPacetylcholinesterase conjugate reagent (cGMP-AChE) competed for binding to cGMP-specific rabbit antiserum. Complexes consisting of cGMP and/or cGMP-AChE and rabbit IgG were captured to a mouse monoclonal anti-rabbit IgG antibody that had been previously coated on the assay plate. The plate was washed and Ellman's Reagent (AChE substrate) was added to the wells. The AChE enzymatic reaction produced a yellow color that was read on a SpectraMax Spectrophotometer (Molecular Devices, Sunnyvale, CA, USA) at $420 \mathrm{~nm}$. The assay signal was proportional to the concentration of cGMP-AChE bound to the well, and inversely proportional to the cGMP concentration in each sample. The relative concentration of cGMP in each sample was determined by interpolation of raw signals using a standard calibrator curve and a four-parameter logistic fit with a weighting factor of $1 / Y$. The LLOQ was $138 \mathrm{pmol} /$ $\mathrm{mL}$ cGMP in neat human urine. Raw urine cGMP concentrations were normalized to the corresponding urine creatinine concentration in each sample for further analysis.

\subsubsection{Relative Quantitative Measurement of Collagen Type $X$ Marker (CXM) in Human Serum}

CXM was measured in serum as an exploratory biomarker of growth plate activity directly related to the effect of vosoritide on endochondral bone growth. In the phase II study, predose serum samples were collected on days 1, 10, 29, 85 and 183 during the initial 6-month period, and at months 8 , $10,12,15,18,21$ and 24 during the extension phase. In the phase III study, samples were collected on day 1 and weeks $13,26,39$ and 52 .

CXM was measured in serum using a validated relative quantitative ECLA (modification of the method described by Coghlan et al. [30]), in the phases II and III studies. 96-well MSD streptavidin plates were blocked with StartingBlock PBS with Tween-20 (ThermoFisher Scientific, Waltham, MA, USA). After decanting blocking buffer, biotinylated anti-human collagen type X NC1 domain capture SOMAmer (SomaLogic, Boulder, CO, USA) was incubated on the plate. The standard stock consisting of recombinant human collagen type X NC1 domain (BioMatik, Kitchener, ON, Canada) in assay diluent (AD) was serially diluted in $\mathrm{AD}$, while serum quality control samples (QCs) and serum study samples were diluted 1:100 in AD. After washing the assay plate, diluted calibrators and samples were incubated on 
the plate. After a second wash, ruthenium-labeled mouse monoclonal anti-collagen type X NC1 domain IgG detection antibody was incubated on the plate. The plate was then washed, MSD Read Buffer T with surfactant was added, and the plate was read on an MSD Quickplex instrument. The raw signal from each well was proportional to the collagen type $\mathrm{X}$ concentration in each sample. The concentration of collagen type $\mathrm{X}$ in each unknown sample was determined by interpolation of the raw assay signal using the standard calibrator curve. The standard regression performed by Watson LIMS used a four-parameter logistic Marquardt model with a weighting factor of $1 / \mathrm{Y}^{2}$. The assay limit of detection was $914 \mathrm{pg} / \mathrm{mL}$ CXM in neat human serum.

\subsubsection{Semi-Quantitative Measurement of Total Anti-Vosoritide Antibodies in Human Serum}

Predose serum samples for immunogenicity assessment were collected at the same visits as CXM samples in the phases II and III studies. Total anti-vosoritide antibody (TAb) titers were measured using a validated semi-quantitative bridging ECLA method. The TAb assay method used bridging of biotin- and ruthenium-conjugated vosoritide reagents to detect anti-vosoritide antibodies of all isotypes in human serum samples. Anti-vosoritide antibodies present in serum samples formed complexes with conjugated vosoritide reagents in solution. The mixture was then incubated on a streptavidin-coated assay plate to capture vosoritide antibody complexes. After a final wash, MSD Read Buffer T with surfactant was added and raw assay signals were read on an MSD Quickplex instrument.

Samples that produced assay signal greater than or equal to the screening cut point (statistically defined at the upper 95th percentile of signals from pretreatment study samples) after a 1:10 dilution were considered reactive in an initial screening assay. Samples that screened positive were confirmed positive in a second-tier assay if the signal inhibition in the presence of free vosoritide exceeded the confirmation cut point established during method validation. A semi-quantitative titer value was determined for confirmed positive samples in a third-tier assay by serially diluting each sample until the signal crossed the titer cut point. The interpolated dilution factor at which the signal of the dilution curve crossed the titer cut point was reported as the sample titer. The assay limit of detection was $37.5 \mathrm{ng} / \mathrm{mL}$ anti-vosoritide antibody in neat serum.

\subsubsection{Semi-Quantitative Measurement of Vosoritide-Neutralizing Antibodies in Human Serum}

Study samples that were confirmed positive for antivosoritide $\mathrm{TAb}$ were further analyzed for anti-vosoritide neutralizing antibodies (NAbs) capable of interfering with vosoritide signaling through the cell-surface receptor NPR-B using a validated cell-based assay. Frozen ready-to-assay $\mathrm{NIH} / 3 \mathrm{~T} 3$ cells were seeded in 96-well culture plates with D10 media (Dulbecco's Modified Eagle's Medium [high glucose $]+10 \%$ fetal bovine serum) and cultured overnight in a $5 \% \mathrm{CO}_{2}$ incubator at $37^{\circ} \mathrm{C}$. After overnight culture, the cells were stimulated with $5 \mathrm{ng} / \mathrm{mL}$ vosoritide in the presence of diluted human serum samples. Vosoritide NAbs in samples interfered with the activation of cellular NPR$\mathrm{B}$, reducing the amount of intracellular cGMP generated. After stimulation, cells were lysed and cGMP concentrations in cell lysates were measured using a competitive format ELISA kit from Cayman Chemical Co. (Ann Arbor, MI, USA; described above). The raw assay signal in the cGMP ELISA was inversely proportional to the cGMP concentration in each sample. Therefore, samples containing NAbs generated higher assay signals due to reduced cellular cGMP production.

Samples that produced absorbance at or above the established screening cut point (set at the upper 95th percentile of pretreatment sera) were considered potentially positive and were further tested in the confirmation step, in which antivosoritide antibodies were removed by immunodepletion using vosoritide-conjugated magnetic beads. Samples with \%inhibition after immunodepletion that was greater than or equal to the confirmation cut point defined in the validation were considered positive. Samples that were screened and confirmed positive were serially diluted and assayed to determine a semi-quantitative titer value. The interpolated dilution factor at which the signal of the dilution curve crossed the titer cut point was reported as the sample titer. The assay limit of detection was $227 \mathrm{ng} / \mathrm{mL}$ anti-vosoritide $\mathrm{IgG}$ in human serum.

\subsection{Pharmacokinetic Analysis}

Plasma vosoritide PK parameters, including $C_{\max }$, $T_{\max }, \mathrm{AUC}_{0-\mathrm{t}}$, AUC from time zero to $60 \mathrm{~min}$ postdose $\left(\mathrm{AUC}_{0-60 \mathrm{~min}}\right), \mathrm{AUC}$ from time zero to infinity $\left(\mathrm{AUC}_{0-\infty}\right)$ and elimination half-life $\left(t_{1 / 2}\right)$, were obtained using standard non-compartmental analysis in the phase II and III studies. AUC values were calculated using the linear trapezoidal rule for increasing concentrations and the logarithmic trapezoidal rule for decreasing concentrations. Where appropriate, individual mean $C_{\max }$ and $\mathrm{AUC}_{0-\mathrm{t}}$ or $\mathrm{AUC}_{0-60 \min }$ values were calculated for each patient, using available $\mathrm{PK}$ data from all visit days, and were used as representative measures of a patient's exposure during the respective durations. Plasma vosoritide concentrations that were below the LLOQ at timepoints prior to $T_{\max }$ were treated as zero, while those that were below the LLOQ at timepoints after $T_{\max }$ were censored (set to 'missing'). 


\subsection{Analysis of Factors Affecting Vosoritide Pharmacokinetics}

The impact of patient demographics, including sex, body weight, age and race, as well as the development of antivosoritide antibody (ADA), on plasma vosoritide exposure were evaluated. The potential relationships between vosoritide exposure and body weight and age were investigated using linear regressions to determine if the slopes of the relationships were significantly different from zero at a significance level of 0.05 . The distribution of vosoritide exposures between each category of sex, race and ADA were compared to determine the impact of each factor on vosoritide exposure. The significance of the impact of $\mathrm{TAb}$ on the PK parameters was tested using a $t$ test.

\subsection{Analysis of Pharmacokinetics and Efficacy}

The primary efficacy endpoint is the change from baseline in AGV. For a given interval (Date 1 to Date 2), AGV is defined as follows (Eq. 1): vosoritide exposure obtained at $15 \mu \mathrm{g} / \mathrm{kg}$ and changes in AGV from baseline to week 52. This would indicate that the plateau of the exposure-efficacy curve has been reached.

\subsection{Analysis of Pharmacokinetics and Biomarkers}

For the phase II study, potential correlations between vosoritide exposure across the $2.5-30 \mu \mathrm{g} / \mathrm{kg}$ dose range, and mean CXM from the day 10 (the first on-treatment assessment) to day 183 phase were explored using a sigmoid $E_{\max }$ model (Eq. 3),

$\overline{\mathrm{CXM}}=E_{0}+\frac{\mathrm{PK}^{h} \times E_{\max }}{\mathrm{PK}^{h}+\mathrm{EC}_{50}^{h}}$,

where $\overline{\mathrm{CXM}}$ is the individual mean CXM from day 10 to day $183, P K$ is the individual mean $\mathrm{PK}$ parameter $\left(C_{\max }\right.$ or $\left.\mathrm{AUC}_{0-t}\right)$, $E_{0}$ is the baseline level, $E_{\max }$ is the maximum possible pharmacologic effect on $\mathrm{CXM}, \mathrm{EC}_{50}$ is the $\mathrm{PK}$ parameter value producing half maximal effect, and $h$ is the Hill coefficient. Data from patients administered 15 or $30 \mu \mathrm{g} / \mathrm{kg}$ from day 10

$\operatorname{AGV}(\mathrm{cm} / \mathrm{yr})=\frac{\text { Standing height at Date } 2-\text { Standing Height at Date } 1}{\text { Date } 2-\text { Date } 1 \text { (days) }} \times 365.25$.

The baseline AGV was established based on data of the last 6 months in the observational study (ClinicalTrials.gov NCT01603095; date of registration, 22 May 2012).

For the phase II study, potential correlations between vosoritide exposure and change in AGV from baseline to day 183 were explored, during which patients were administered daily subcutaneous injections of 2.5 (Cohort 1), 7.5 (Cohort 2), 15 (Cohort 3) or $30 \mu \mathrm{g} / \mathrm{kg}$ (Cohort 4), and from baseline to month 24 for Cohorts $3(15 \mu \mathrm{g} / \mathrm{kg})$ and $4(30 \mu \mathrm{g} /$ $\mathrm{kg})$. Data used in the phase II study from baseline to day 183 were modeled using a sigmoid maximum effect $\left(E_{\max }\right)$ model (Eq. 2),

$\Delta \mathrm{AGV}=E_{0}+\frac{\mathrm{PK}^{h} \times E_{\max }}{\mathrm{PK}^{h}+\mathrm{EC}_{50}^{h}}$,

where $\triangle \mathrm{AGV}$ is the change in $\mathrm{AGV}$ from baseline to day 183 , $\mathrm{PK}$ is the individual mean $\mathrm{PK}$ parameter $\left(C_{\max }\right.$ or $\left.\mathrm{AUC}_{0-\mathrm{t}}\right)$, $E_{0}$ is the baseline effect, $E_{\max }$ is the maximum possible effect, $\mathrm{EC}_{50}$ is the PK parameter value producing half maximal effect, and $h$ is the Hill coefficient. Exposure data from patients administered 15 or $30 \mu \mathrm{g} / \mathrm{kg}$ from baseline to month 24 were then modeled using linear regression to determine if the slope of the relationship was significantly different from zero at a significance level of 0.05 .

Data used in the phase III study were also modeled using linear regression to confirm the lack of correlation between to month 24 were then modeled using linear regression to determine if the slope of the relationship was significantly different from zero at a significance level of 0.05 .

Data used in the phase III study were also modeled using linear regression to confirm the lack of correlation between vosoritide exposure obtained at $15 \mu \mathrm{g} / \mathrm{kg}$ and mean CXM from week 13 to week 52 . This would indicate that the plateau of the exposure-CXM curve has been reached.

Besides CXM, for the phase II study, potential correlations between vosoritide exposure across the $2.5-30 \mu \mathrm{g} / \mathrm{kg}$ dose range and visit-matched maximum postdose increases in urine cGMP normalized to creatinine (cGMP/Cr) over the predose level at each visit were evaluated for the initial 6-month period. The predose cGMP/Cr assessment at a given visit was used as the baseline value to calculate the maximum postdose increase at that visit. In cases where cGMP/Cr was maximal at predose, the maximum postdose increase was represented as a negative value. Individual $C_{\max }$ and AUC that were not estimable due to samples being below the limit of quantitation were set to zero for these analyses.

Data from the phase II study were modeled using a sigmoid $E_{\max }$ model (Eq. 4):

$\Delta \mathrm{cGMP}=E_{0}+\frac{\mathrm{PK}^{h} \times E_{\max }}{\mathrm{PK}^{h}+\mathrm{EC}_{50}^{h}}$, 
where $\Delta c \mathrm{cMMP}$ is the maximum increase in $\mathrm{cGMP} / \mathrm{Cr}$, $\mathrm{PK}$ is the visit-matched $\mathrm{PK}$ parameter $\left(C_{\max }, \mathrm{AUC}_{0-\mathrm{t}}\right.$ or $\left.\mathrm{AUC}_{0-60 \min }\right)$, $E_{0}$ is the baseline level, $E_{\max }$ is the maximum possible pharmacologic effect on cGMP/Cr, $\mathrm{EC}_{50}$ is the $\mathrm{PK}$ parameter value producing half maximal effect, and $h$ is the Hill coefficient. Data from the phase III study were modeled using an $E_{\max }$ model, where $h$ is 1 .

\subsection{Analysis of Pharmacokinetics and Heart Rate as Well as Systolic and Diastolic Blood Pressures}

Potential relationships between vosoritide exposure and maximum changes from predose heart rate as well as systolic and diastolic blood pressures were evaluated in the phases II and III studies using linear regressions to determine if the slopes of the relationships were significantly different from zero at a significance level of 0.05 .

\subsection{Software}

Pharmacokinetic parameters were estimated using noncompartmental analysis using a validated version of Phoenix WinNonlin (version 6.4 or later; Pharsight Corporation, Mountain View, CA, USA). Data processing and further analyses were performed using $\mathrm{R}$ (version 3.4.1 or later; The R Foundation for Statistical Computing, Vienna, Austria) with R Studio (version 1.1.423 or later; R Studio, Inc., Boston, MA, USA).

\section{Results}

\subsection{Vosoritide Pharmacokinetics in Children with Achondroplasia}

The mean single-dose PK profiles obtained from the phase II study and the mean PK profiles across visits obtained from the phase III study are shown in Fig. 1. Key PK parameters from the phase II study are described earlier and are summarized in Table 1. After 52 weeks of treatment in the phase III study, median $T_{\max }$ was 15 min and mean $t_{1 / 2}$ was 27.9 min; mean $C_{\max }$ and $\mathrm{AUC}_{0-\infty}$ were $5800 \mathrm{pg} / \mathrm{mL}$ and $276,000 \mathrm{pg}-\mathrm{min} / \mathrm{mL}$, respectively. Mean apparent clearance $(\mathrm{CL} / F)$ and apparent volume of distribution $\left(V_{\mathrm{z}} / F\right)$ were 79.4 $\mathrm{mL} / \mathrm{min} / \mathrm{kg}$ and $2910 \mathrm{~mL} / \mathrm{kg}$, respectively (Table 2). Plasma vosoritide exposure in treated patients was consistent over 52 weeks and was comparable with that observed in patients administered the $15 \mu \mathrm{g} / \mathrm{kg}$ dose in the phase II study, as previously reported [33]. Results from the phase III study also suggest that plasma vosoritide $\mathrm{AUC}_{0-60 \mathrm{~min}}$ is positively correlated with body weight and age $(p<0.001)$ [Fig. S1 in the electronic supplementary material (ESM)], while the sex of a patient had no apparent impact on plasma vosoritide $C_{\max }$ or $\mathrm{AUC}_{0-60 \min }$ (Fig. S2 in the ESM). The impact of race on vosoritide exposure was also evaluated but the data were insufficient to draw conclusions (Fig. S3 in the ESM).

\subsection{Impact of Immunogenicity on Vosoritide Pharmacokinetics}

In the phase III study, TAb responses were detected in the serum of $42 \%(25 / 60)$ of treated patients, and all placebotreated patients tested negative for $\mathrm{TAb}$ at all time points tested. All of the TAb-positive patients tested negative for $\mathrm{NAb}$ at all time points tested. Vosoritide plasma exposures $\left(C_{\max }\right.$ and $\left.\mathrm{AUC}_{0-\infty}\right)$ were comparable between visits where there was detectable TAb titer and where there was no detectable TAb titer $(p>0.8$ for the difference in estimated mean $C_{\max }$ and $\mathrm{AUC}_{0-\infty}$ by TAb status tested with a $t$-test), suggesting there was no impact of serum TAb on plasma exposure of vosoritide (Fig. 2).

\subsection{Pharmacokinetics and Annualized Growth Velocity}

In the phase II study, sigmoid $E_{\max }$ model parameter estimates with 95\% CIs for the PK-AGV relationship are provided in Table 3. Analysis of the phase II data demonstrated a steep exposure-response relationship between the range of exposures obtained with the $2.5 \mu \mathrm{g} / \mathrm{kg}$ through $7.5 \mu \mathrm{g} /$ $\mathrm{kg}$ doses $\left(h=2.26\right.$ for $\left.\mathrm{AUC}_{0-\mathrm{t}}\right)$. This was followed by a relatively similar response at the exposure range obtained with the 15 and $30 \mu \mathrm{g} / \mathrm{kg}$ doses (Fig. 3a). There was no significant correlation between vosoritide plasma exposure and change in AGV through 24 months of daily dosing across the exposure range obtained with daily doses between 15 and $30 \mu \mathrm{g} / \mathrm{kg}\left(p=0.34\right.$ for $C_{\max }$, and $p=0.50$ for $\left.\mathrm{AUC}_{0-60 \min }\right)$ [Fig. 3b], suggesting the exposures obtained at these dose levels yielded a maximal and saturated response, with no additional meaningful improvements in AGV at $30 \mu \mathrm{g} / \mathrm{kg}$ compared with $15 \mu \mathrm{g} / \mathrm{kg}$.

The data from the phase III study further confirmed the change in AGV from baseline had reached the plateau of the exposure-response curve at exposures obtained at $15 \mu \mathrm{g} / \mathrm{kg}$ (Fig. 3c), as evidenced by the lack of relationship between exposure and changes in $\mathrm{AGV}$ in patients receiving $15 \mu \mathrm{g} / \mathrm{kg}$ vosoritide ( $p=0.52$ for $C_{\max }$, and $p=0.54$ for $\left.\mathrm{AUC}_{0-60 \mathrm{~min}}\right)$.

\subsection{Pharmacokinetics and Biomarkers}

Transient increases in urine cGMP from predose levels, during a dosing interval, were observed following vosoritide administration to patients with achondroplasia. The phase II study showed that the maximum postdose increase in urine cGMP/Cr over the predose level at each visit was dosedependent, and the magnitude of the maximal increases at 
Fig. 1 Mean (+SD) concentration-time profiles of vosoritide in plasma observed in the phases II and III studies. a Mean (+SD) concentration-time profiles of vosoritide in plasma observed on day 1 in the phase II study, color-coded by study cohort and dose (C1: Cohort $1,2.5 \mu \mathrm{g} / \mathrm{kg}$; C2: Cohort 2, 7.5 $\mu \mathrm{g} / \mathrm{kg}$; C3: Cohort 3, $15 \mu \mathrm{g} / \mathrm{kg}$; C4: Cohort 4, $30 \mu \mathrm{g} / \mathrm{kg}$ ). Each line represents the mean plasma vosoritide concentration across patients receiving $2.5,7.5$, 15.0 or $30.0 \mu \mathrm{g} / \mathrm{kg}$ vosoritide on day 1. Error bars represent the standard deviation of the mean, and only the upper error bars are shown. b Mean $(+\mathrm{SD})$ concentration-time profiles of vosoritide in plasma observed at $15 \mu \mathrm{g} / \mathrm{kg}$ in the phase III study, color-coded by visits. Each line represents the mean plasma vosoritide concentration across patients for each visit. Error bars represent the standard deviation of the mean, and only the upper error bars are shown. The LLOQ for the PK assays used in the phases II and III studies were $391 \mathrm{pg} / \mathrm{mL}$ and $137 \mathrm{pg} / \mathrm{mL}$ in neat plasma, respectively. $S D$ standard error, $L L O Q$ lower limit of quantitation, $P K$ pharmacokinetic
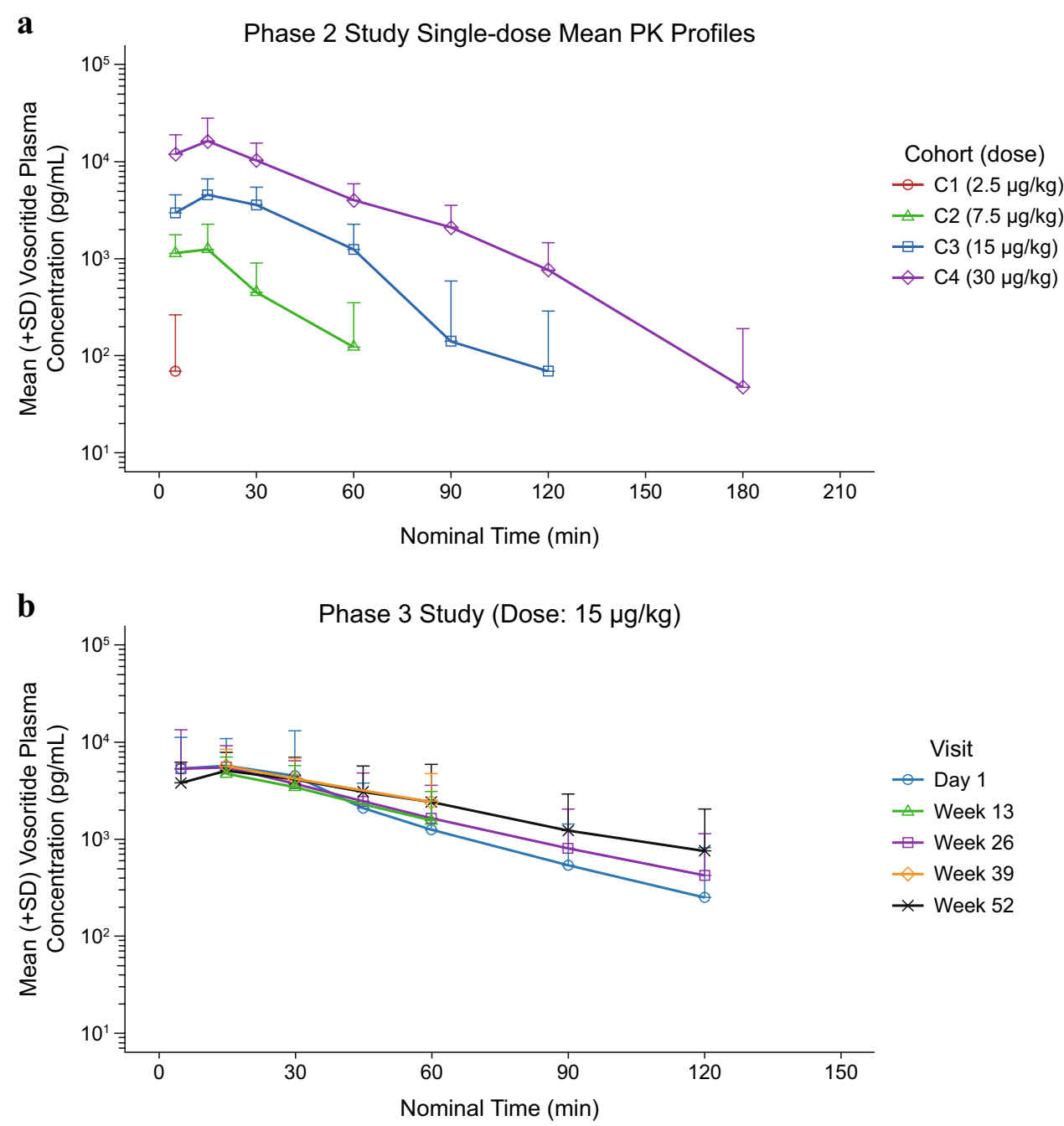

each dose remained similar over 24 months of treatment in patients administered 15 or $30 \mu \mathrm{g} / \mathrm{kg}$ [33], suggesting vosoritide treatment induced sustained pharmacological activity through binding to the NPR-B receptor with each dose over 24 months of treatment. The phase II study also showed an increase in the median CXM concentrations above baseline, which remained elevated across 24 months, suggesting vosoritide treatment at $15-30 \mu \mathrm{g} / \mathrm{kg}$ induced a sustained increase in growth plate activity. Moreover, median serum CXM concentrations were also similar in patients administered $15 \mu \mathrm{g} /$ $\mathrm{kg}$ as those administered $30 \mu \mathrm{g} / \mathrm{kg}$, indicating a maximal CXM response at exposures obtained at $15 \mu \mathrm{g} / \mathrm{kg}$ [33].

In the phase II study, sigmoid $E_{\max }$ model parameter estimates with $95 \%$ CIs for the PK-CXM relationship are provided in Table 4. Like AGV, analysis of the phase II data demonstrated a steep exposure-response relationship between the mean CXM and the range of exposures obtained with the $2.5 \mu \mathrm{g} / \mathrm{kg}$ through $7.5 \mu \mathrm{g} / \mathrm{kg}$ doses $(h=3.93$ for $C_{\max }$, and $h=1.10$ for $\mathrm{AUC}_{0-\mathrm{t}}$ ), and an upper plateau of mean CXM at exposure observed at 15 and $30 \mu \mathrm{g} / \mathrm{kg}$ dose levels (Fig. 4a). There was also no significant correlation between vosoritide plasma exposure and mean CXM concentration through 24 months of daily dosing across the exposure range obtained with daily doses between 15 and $30 \mu \mathrm{g} / \mathrm{kg}\left(p=0.98\right.$ for $C_{\max }$, and $p=0.99$ for $\left.\mathrm{AUC}_{0-60 \mathrm{~min}}\right)$ (Fig. 4b), suggesting the exposures obtained with daily doses $\geq 15 \mu \mathrm{g} / \mathrm{kg}$ yielded a maximal and saturated CXM response.

In the phase III study, transient increases in urine cGMP/ $\mathrm{Cr}$ were also observed in the treated group and were similar over 52 weeks, while remaining unchanged in the placebo group (Fig. 5). Increases in the mean and median CXM concentrations above baseline were also observed in patients in the treated group by week 13 (first on-treatment assessment) and were sustained through 52 weeks, while CXM concentrations remained relatively unchanged in patients in the placebo group (Fig. 6). The data from the phase III study further confirmed that CXM concentrations had reached the plateau of the exposure-CXM curve at exposures obtained at 
Table 2 Summary of vosoritide PK parameters in treated patients in the phase III study

\begin{tabular}{|c|c|c|c|c|c|c|c|c|c|}
\hline Visit & Statistic & $T_{\max }(\min )$ & $C_{\max }(\mathrm{pg} / \mathrm{mL})$ & $\begin{array}{l}\mathrm{AUC}_{0-60 \min } \\
(\mathrm{pg}-\mathrm{min} / \mathrm{mL})\end{array}$ & $\mathrm{AUC}_{0-\mathrm{t}}(\mathrm{pg}-\mathrm{min} / \mathrm{mL})$ & $\begin{array}{l}\mathrm{AUC}_{0-\infty} \\
\text { (pg-min/ } \\
\mathrm{mL})\end{array}$ & $\begin{array}{l}\mathrm{CL} / \mathrm{F}(\mathrm{mL} / \\
\mathrm{min} / \mathrm{kg})\end{array}$ & $V_{\mathrm{z}} / F(\mathrm{~mL} / \mathrm{kg})$ & $t_{1 / 2}(\min )$ \\
\hline \multirow[t]{3}{*}{ Day 1} & Mean & 13.8 & 7180 & 208,000 & 242,000 & 244,000 & 104 & 2880 & 21.0 \\
\hline & SD & 7.23 & 9650 & 198,000 & 232,000 & 224,000 & 98.8 & 2450 & 4.67 \\
\hline & $\mathrm{CV} \%$ & 52.4 & 134 & 95.2 & 95.8 & 91.8 & 95.2 & 85.1 & 22.2 \\
\hline \multirow[t]{3}{*}{ Week 26} & Mean & 14.5 & 6520 & 205,000 & 256,000 & 283,000 & 85.2 & 3020 & 26.6 \\
\hline & SD & 7.62 & 7890 & 151,000 & 211,000 & 249,000 & 53.2 & 1980 & 9.08 \\
\hline & CV\% & 52.6 & 121 & 73.5 & 82.6 & 87.9 & 62.5 & 65.7 & 34.2 \\
\hline \multirow[t]{3}{*}{ Week 52} & Mean & 16.8 & 5800 & 212,000 & 290,000 & 276,000 & 79.4 & 2910 & 27.9 \\
\hline & SD & 11.7 & 3680 & 133,000 & 235,000 & 187,000 & 53.0 & 1660 & 9.91 \\
\hline & CV\% & 69.6 & 63.4 & 62.9 & 81.2 & 67.5 & 66.8 & 57.1 & 35.5 \\
\hline
\end{tabular}

All summary statistics rounded to three significant figures

Two patients discontinued from treatment on day 7 and day 15, respectively. Their PK data were available for day 1 but not for later visits

On day 1, one patient was administered a dose higher than the nominal dose and the associated PK were excluded from summary statistics. Another subject had no PK parameters as the actual sampling times were negative for all PK samples

On week 26, two patients had no PK parameters as only predose PK samples were taken. One patient had no PK parameters as two PK samples were associated with the same actual sampling time of $15 \mathrm{~min}$

On week 52, PK samples were not taken for two patients

$A U C_{0-\infty}$ area under the plasma concentration-time curve from time zero to infinity, $A U C_{0-t}$ area under the plasma concentration-time curve from time zero to the time of the last measurable concentration, $A U C_{0-60 \min }$ area under the plasma concentration-time curve from time zero to 60 min postdose, $C L / F$ apparent clearance, $C_{\max }$ maximum observed plasma concentration, $C V$ coefficient of variation, $P K$ pharmacokinetic, $S D$ standard deviation, $t_{1 / 2}$ half-life, $T_{\max }$ time to reach maximum concentration, $V_{z} / F$ apparent volume of distribution

$15 \mu \mathrm{g} / \mathrm{kg}$ (Fig. 4c), as evidenced by the lack of relationship between exposure and mean CXM concentrations in patients receiving $15 \mu \mathrm{g} / \mathrm{kg}$ vosoritide ( $p=0.74$ for $C_{\max }$, and $p=$ 0.58 for $\left.\mathrm{AUC}_{0-60 \mathrm{~min}}\right)$.

$E_{\max }$ model parameter estimates with $95 \%$ CIs for the PK-cGMP relationships for the initial 6-month period of the phases II and III studies are provided in Tables 5 and 6 , respectively. Unlike AGV and the CXM biomarker, exposure-cGMP analyses showed that systemic pharmacological activity is near maximal or saturated at exposures obtained at the highest dose studied (i.e. $30 \mu \mathrm{g} / \mathrm{kg}$ ) [Fig. 7]. The mean $\mathrm{AUC}_{0 \text {-t }}$ observed with $30 \mu \mathrm{g} / \mathrm{kg}$ in the initial 6 months of the phase II study $\left(1.27 \times 10^{6} \mathrm{pg}-\mathrm{min} / \mathrm{mL}\right)$ was approximately sixfold greater than the estimated $E C_{50}\left(2.22 \times 10^{5}\right.$ $\mathrm{pg}-\mathrm{min} / \mathrm{mL}$ ). Given that exposure-response relationships for changes in both AGV and the CXM biomarker were saturated at $15 \mu \mathrm{g} / \mathrm{kg}$, the additional systemic pharmacological activity, indicated by the increase in cGMP/Cr at the higher end of the exposure range (i.e. exposure from $30 \mu \mathrm{g} / \mathrm{kg}$ ), was likely present in tissues not related to endochondral bone formation.

\subsection{Pharmacokinetics and Heart Rate as Well as Systolic and Diastolic Blood Pressures}

The relationships between visit-matched vosoritide $C_{\max }$ and the maximum increase from predose heart rate for the initial
6 months of the phase II study and the 52-week duration of the phase III study are shown in Fig. 8. Weak positive correlations between the maximum increase from predose heart rate and exposure were observed and were statistically significant $(p<0.05)$ but not clinically meaningful. Similarly, the relationships between visit-matched vosoritide $C_{\max }$ and the maximum decreases from predose systolic and diastolic blood pressures for each study are shown in Fig. 9. The results showed no correlation between vosoritide plasma exposure and decrease in systolic or diastolic blood pressure ( $p=0.54$ for phase II systolic blood pressure vs. $C_{\max }$ correlation; $p=0.72$ for phase III systolic blood pressure vs. $C_{\max }$ correlation; $p=0.43$ for phase II diastolic blood pressure vs. $C_{\max }$ correlation; and $p=0.79$ for phase III diastolic blood pressure vs. $C_{\max }$ correlation). For each vital sign, the results for the 24-month period of the phase II study showed a similar trend to that of the analysis for the initial 6-month period.

Overall, there were no clinically meaningful correlations between vosoritide plasma exposure and changes from predose heart rate, as well as systolic or diastolic blood pressures in patients with achondroplasia treated with vosoritide. Exposure-response analyses were also conducted for incidences of reported treatmentemergent adverse events based on predefined search criteria for hypotension and injection site reactions in the phase II study, and no meaningful correlations were observed between vosoritide plasma exposure and the reporting of either hypotension or injection site reactions (Figs. S10 and S11 in the ESM). 


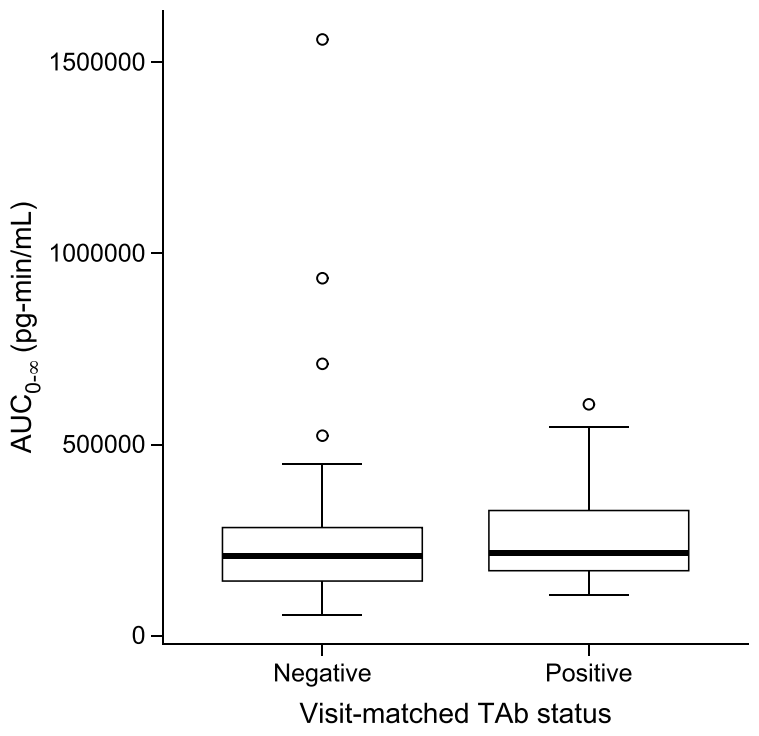

Fig. 2 Distribution of individual mean vosoritide $\mathrm{AUC}_{0-\infty}$ in plasma by serum TAb status (negative or positive) in the phase III study. The line inside the box represents the median and the box represents the limits of the middle half of the data. The range of the box, from the first quartile (Q1) to the third quartile (Q3), defines the IQR. The standard span of the data is defined within the range from Q1 - 1.5 $\times \mathrm{IQR}$ to $\mathrm{Q} 3+1.5 \times \mathrm{IQR}$. Whiskers are drawn to the nearest value not beyond the range of the standard span; points beyond are drawn as individual open circles. Distribution of the individual mean vosoritide $C_{\max }$ is similar and is shown in Fig. S4 of the ESM. $A U C_{0-\infty}$ area under the plasma concentration-time curve from time zero to infinity, $T A b$ total anti-vosoritide antibody, $I Q R$ interquartile range, $C_{\max }$ maximum observed plasma concentration, ESM electronic supplementary material

\section{Discussion}

Most peptides have very low oral bioavailability and are often delivered subcutaneously, where they enter systemic circulation through the blood capillaries and/or lymphatic vessels depending on their molecular weight [35]. They are also rapidly cleared from systemic circulation through enzymatic proteolysis and/or renal filtration, resulting in very short half-lives of a few minutes [36]. While endogenous CNP binds to the NPR-B receptor for intracellular signaling, it is predominately cleared by intracellular proteases through binding with the NPR-C receptor and the extracellular NEP protease, resulting in a short half-life of $2.6 \mathrm{~min}$ in humans [37]. The structural modification of vosoritide confers resistance to proteolysis by NEP, such that its half-life is extended almost tenfold in comparison with CNP. This increases exposure to the target growth plate and allows for once-daily subcutaneous administration to produce its desired pharmacologic effect.

Nonlinear PK have been observed in biologics, including peptides, and have been attributed to target-mediated drug disposition (TMDD) [38]. While the PK profiles for vosoritide do not indicate that TMDD is at play, the observed greater than proportional increase in vosoritide exposure with doses from 2.5 to $30 \mu \mathrm{g} / \mathrm{kg}$ may result from the saturation of binding sites. While the NPR-B and NPR-C receptors are both expressed in many organs [29, 39], these binding sites may become saturated when the concentration of vosoritide is higher relative to the number of available binding sites in situ. The lack of accumulation is supported by the consistent vosoritide plasma exposure ( $C_{\max }$ and AUC) observed over 52 weeks of the phase III study. This is consistent with the short half-life of vosoritide relative to the once-daily dosing.

The development of ADA may impact the PK and PD of exogenously administered therapeutic proteins, including peptides. A therapeutic protein may bind with clearing ADA to form immune complexes and the elimination of these complexes increases the clearance of the protein, leading to a shorter half-life. Conversely, sustaining ADA may bind with the therapeutic protein to form complexes but these complexes could either act like storage depots for the protein or recycled through the neonatal Fc receptor, both of which reduce the clearance of the protein, resulting in a longer halflife and higher systemic exposure [40]. Immune responses may also affect the pharmacological activity of therapeutic
Table 3 Sigmoid $E_{\max }$ model parameter estimates for annualized growth velocity (initial 6 months of the phase II study)

\begin{tabular}{|c|c|c|c|c|}
\hline $\begin{array}{l}\text { Pharmacoki- } \\
\text { netic parameter }\end{array}$ & $\begin{array}{l}E_{0}(\mathrm{~cm} / \text { year }) \\
\operatorname{MPE}(95 \% \mathrm{CI})\end{array}$ & $\begin{array}{l}E_{\max }(\mathrm{cm} / \text { year }) \\
\text { MPE }(95 \% \mathrm{CI})\end{array}$ & $\begin{array}{l}E C_{50} \\
\operatorname{MPE}(95 \% \mathrm{CI})\end{array}$ & $\begin{array}{l}h \\
\text { MPE (95\% CI) }\end{array}$ \\
\hline$C_{\max }$ & $-0.282(-1.84$ to 1.28$)$ & $2.80(0.819-4.78)$ & $\begin{array}{l}1500 \mathrm{pg} / \mathrm{mL}(- \\
2920 \text { to } 5920)\end{array}$ & $\mathrm{NA}^{\mathrm{a}}$ \\
\hline $\mathrm{AUC}_{0-\mathrm{t}}$ & -0.311 ( -1.69 to 1.07$)$ & $2.41(0.729-4.10)$ & $\begin{array}{l}20,500 \mathrm{pg}-\mathrm{min} / \\
\mathrm{mL}(-16,000 \\
\text { to } 57,000)\end{array}$ & $2.26(-5.48$ to 10.0$)$ \\
\hline
\end{tabular}

$C_{\text {max }}$ maximum observed plasma concentration, $A U C_{0-t}$ area under the plasma concentration-time curve from time zero to the time of the last measurable concentration, $C I$ confidence interval, $E_{0}$ baseline effect, $E C_{50}$ pharmacokinetic parameter value producing half maximal effect, $E_{\max }$ maximum possible effect, $h$ Hill coefficient, $M P E$ model parameter estimates, $N A$ not available

${ }^{a}$ Data did not support estimation of $h$ for $C_{\max }$; data were modeled using the $E_{\max }$ model (i.e. $h=1$ ) 

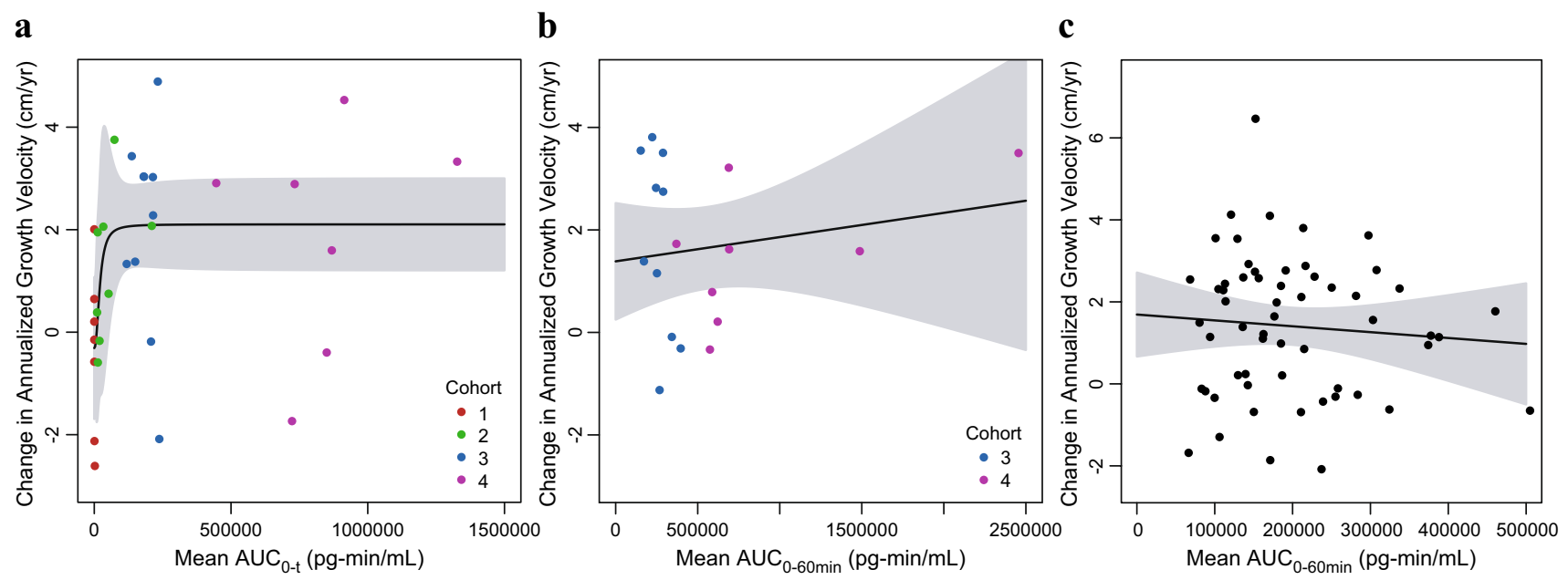

Fig. 3 Change in annualized growth velocity from baseline by individual mean AUC in the phase II and III studies. a Points represent the individual change in annualized growth velocity from baseline to day 183 of the phase II study and individual mean vosoritide $\mathrm{AUC}_{0-\mathrm{t}}$, color-coded by study cohort (C1: Cohort $1,2.5 \mu \mathrm{g} / \mathrm{kg}$; C2: Cohort 2, $7.5 \mu \mathrm{g} / \mathrm{kg}$; C3: Cohort 3, $15 \mu \mathrm{g} / \mathrm{kg}$; C4: Cohort 4, $30 \mu \mathrm{g} / \mathrm{kg}$ ). One patient had a high individual mean $\mathrm{AUC}_{0-\mathrm{t}}\left(4.28 \times 10^{6} \mathrm{pg}-\mathrm{min} / \mathrm{mL}\right)$ relative to other patients, but a within-range change in annualized growth velocity $(3.56 \mathrm{~cm} /$ year). This patient was included in the fit through the data, but was not included in this figure. b Points represent the individual change in annualized growth velocity from baseline to month 24 of the phase II study and individual mean vosoritide
$\mathrm{AUC}_{0-60 \min }$, color-coded by study cohort (C3: Cohort 3, $15 \mu \mathrm{g} / \mathrm{kg}$; $\mathrm{C} 4$ : Cohort $4,30 \mu \mathrm{g} / \mathrm{kg}$ ). c Points represent the individual change in annualized growth velocity from baseline from the phase III study and individual mean vosoritide $\mathrm{AUC}_{0-60 \mathrm{~min}}$. Solid lines represent the fits through the data and the shaded regions represent the $95 \%$ confidence intervals. The respective analyses using the individual mean vosoritide $C_{\max }$ are similar and are shown in Fig. S5 in the ESM. AUC area under the plasma concentration-time curve, $A U C_{0-t}$ AUC from time zero to the time of the last measurable concentration, $A U C_{0-60 \min }$ area under the plasma concentration-time curve from time zero to $60 \mathrm{~min}$ postdose, $C_{\max }$ maximum observed plasma concentration, ESM electronic supplementary material

Table 4 Sigmoid $E_{\max }$ model parameter estimates for mean CXM (initial 6 months of the phase II study)

\begin{tabular}{lllll}
\hline $\begin{array}{l}\text { Pharmacokinetic } \\
\text { parameter }\end{array}$ & $\begin{array}{l}E_{0}(\mathrm{pg} / \mathrm{mL}) \\
\text { MPE }(95 \% \mathrm{CI})\end{array}$ & $\begin{array}{l}E_{\max }(\mathrm{pg} / \mathrm{mL}) \\
\text { MPE }(95 \% \mathrm{CI})\end{array}$ & $\begin{array}{l}E C_{50} \\
\text { MPE (95\% CI) }\end{array}$ & $\begin{array}{l}h \\
\text { MPE }(95 \% \text { CI })\end{array}$ \\
\hline$C_{\max }$ & $9270(7000-11,500)$ & $3690(647-6740)$ & $1730 \mathrm{pg} / \mathrm{mL}(-636$ to 4100$)$ & $\begin{array}{l}(-9.93 \\
(-41 \text { to } 16.2)\end{array}$ \\
AUC $_{0-\mathrm{t}}$ & $9040(6530-11,600)$ & $4460(-175$ to 9100$)$ & $\begin{array}{l}5.17 \times 10^{4} \mathrm{pg}-\mathrm{min} / \mathrm{mL}\left(-1.01 \times 10^{5}\right. \\
\left.\text { to } 2.04 \times 10^{5}\right)\end{array}$ & $\begin{array}{l}1.10 \\
(-1.78 \text { to 3.98) }\end{array}$ \\
\hline
\end{tabular}

$C_{\max }$ maximum observed plasma concentration, $A U C_{0-t}$ area under the plasma concentration-time curve from time zero to the time of the last measurable concentration, $C I$ confidence interval, $C X M$ collagen type $\mathrm{X}$ marker, $E_{0}$ baseline level, $E C_{50}$ pharmacokinetic parameter value producing half maximal effect on CXM, $E_{\max }$ maximum possible effect, $h$ Hill coefficient, $M P E$ model parameter estimates

proteins, and, consequently, their safety and efficacy profiles. Immunogenicity analyses were conducted during the phases II and III studies of vosoritide and the results showed that the development of an ADA response to vosoritide was not associated with an increased frequency or severity of hypersensitivity adverse events or injection site reactions, and had no apparent impact on vosoritide PK or efficacy as measured by the change from baseline in AGV $[33,34]$.

The evaluation of growth in children using growth velocity is superior to single static height measurements [41]. This is routinely assessed by measuring heights at 6- or 12 -monthly intervals with stadiometers. Growth velocity is typically highest at birth and decreases to around $6 \mathrm{~cm} / y e a r$ in children with average stature until puberty, when it is accompanied by an acceleration in growth. It then decelerates as the epiphyses fuse and final adult height is reached [41]. In contrast, the expected AGV in children with achondroplasia is about $4 \mathrm{~cm} /$ year [42-44].

While the efficacy and safety of vosoritide have been reported elsewhere $[33,34]$, in this study we present the PK, PD, and exposure-response relationships that confirm the dose selection and treatment effect based on the totality of evidence available. In the phase III study, an increase in urine cGMP/Cr was observed in vosoritide-treated patients, but not in patients in the placebo group, which confirmed the pharmacological activity of vosoritide. CXM concentrations above baseline were also observed in patients in the treated group by week 13 (first on-treatment assessment) 
a

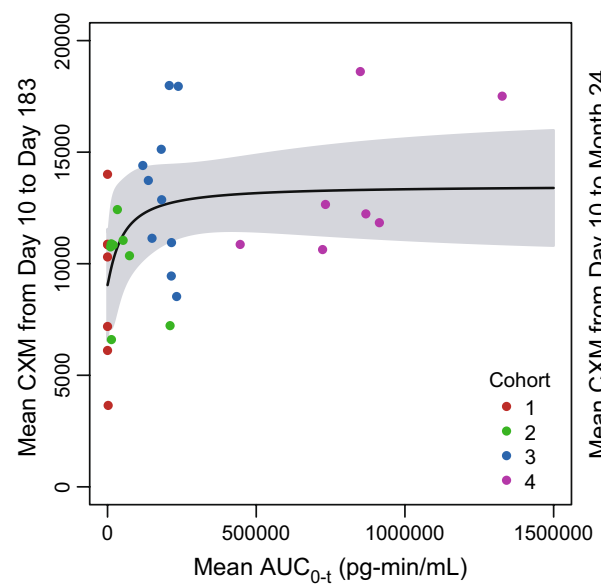

b

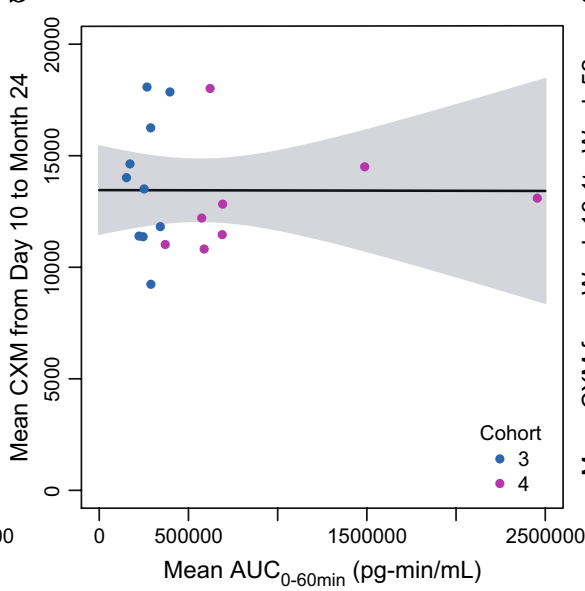

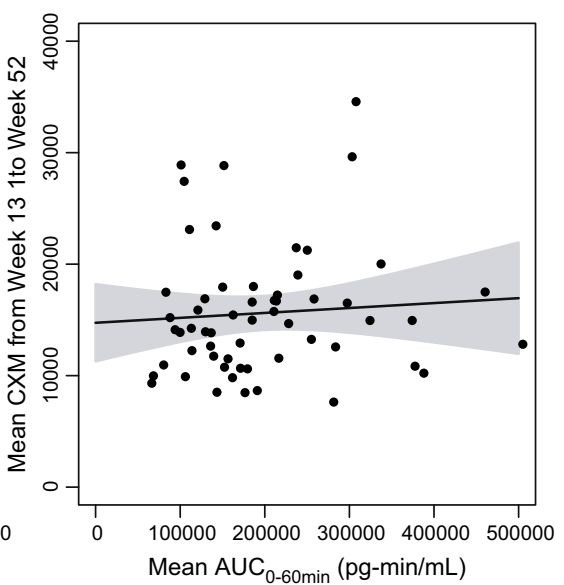

Fig. 4 Change in mean CXM by individual mean AUC in the phase II and III studies. (a) Points represent the individual mean CXM from day 10 to day 183 of the phase II study and individual mean vosoritide $\mathrm{AUC}_{0-\mathrm{t}}$, color-coded by study cohort $(\mathrm{C} 1$ : Cohort $1,2.5 \mu \mathrm{g} / \mathrm{kg}$; C2: Cohort 2, $7.5 \mu \mathrm{g} / \mathrm{kg}$; C3: Cohort 3, $15 \mu \mathrm{g} / \mathrm{kg}$; C4: Cohort 4, 30 $\mu \mathrm{g} / \mathrm{kg})$. One patient had a high individual mean $\mathrm{AUC}_{0-\mathrm{t}}\left(4.28 \times 10^{6}\right.$ $\mathrm{pg}-\mathrm{min} / \mathrm{mL}$ ) relative to other patients, but a within-range mean CXM $(12,200 \mathrm{pg} / \mathrm{mL})$. This patient was included in the fit through the data but was not included in this figure. b Points represent the individual mean CXM from day 10 to month 24 of the phase II study and individual mean vosoritide $\mathrm{AUC}_{0-60 \mathrm{~min}}$, color-coded by study cohort (C3:
Cohort 3, $15 \mu \mathrm{g} / \mathrm{kg}$; C4: Cohort 4, $30 \mu \mathrm{g} / \mathrm{kg}$ ). c Points represent the individual mean CXM from week 13 to week 52 in the phase III study and individual mean vosoritide $\mathrm{AUC}_{0-60 \mathrm{~min}}$. Solid lines represent the fits through the data and the shaded regions represent the 95\% confidence intervals. The respective analyses using the individual mean vosoritide $C_{\max }$ are similar and are shown in Fig. S6 in the ESM. $A U C_{0-t}$ area under the plasma concentration-time curve from time zero to the time of the last measurable concentration, $A U C_{0-60 \mathrm{~min}}$ area under the plasma concentration-time curve from time zero to 60 min postdose, $C X M$ collagen type $\mathrm{X}$ marker, $C_{\max }$ maximum observed plasma concentration, ESM electronic supplementary material

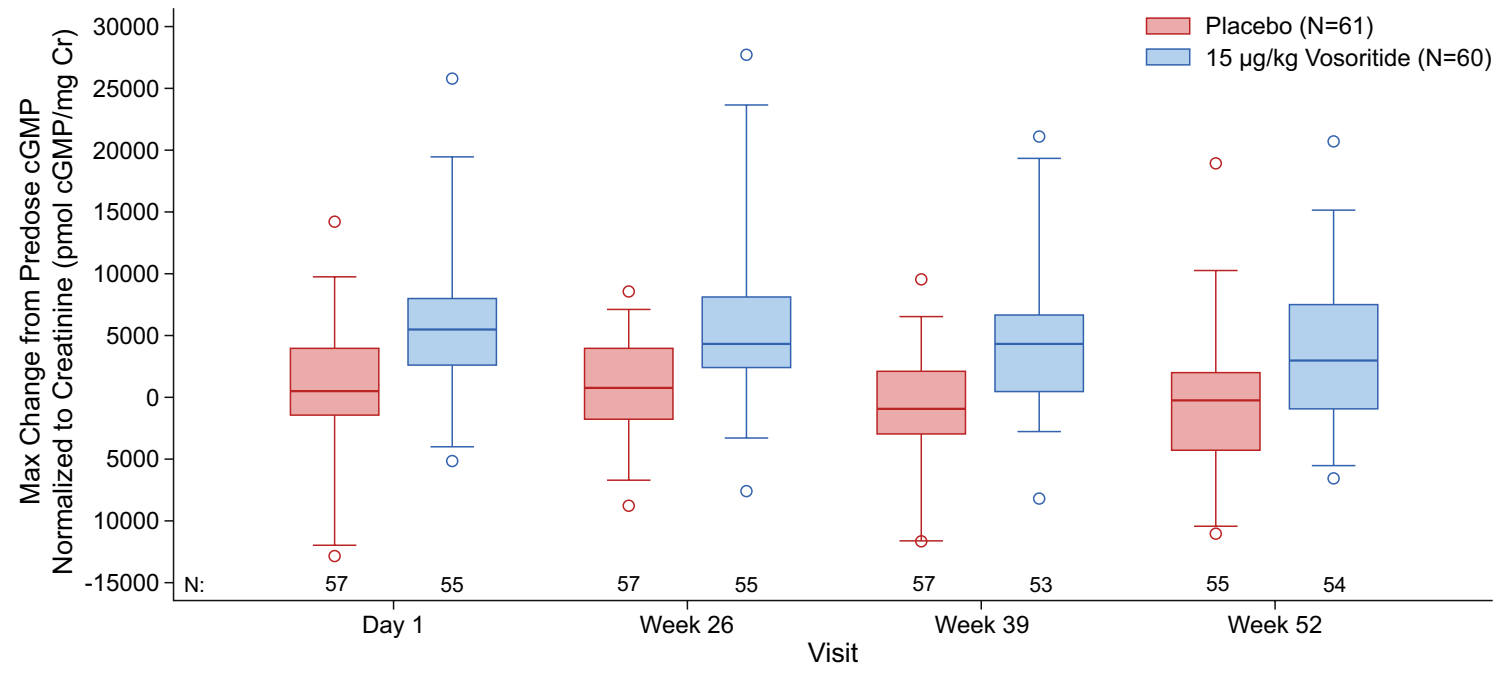

Fig. 5 Distribution of the change from predose to maximum postdose urine cGMP/Cr levels observed in the phase III study. The line inside the box represents the median and the box represents the limits of the middle half of the data. The range of the box, from the first quartile (Q1) to the third quartile (Q3), defines the IQR. The standard span of

and were sustained through 52 weeks, while CXM concentrations remained relatively unchanged in patients in the placebo group, suggesting a greater rate of endochondral ossification in vosoritide-treated patients. the data is defined within the range from $\mathrm{Q} 1-1.5 \times \mathrm{IQR}$ to $\mathrm{Q} 3+1.5$ $\times$ IQR. Whiskers are drawn to the nearest value not beyond the range of the standard span; points beyond are drawn as individual open circles. $c G M P$ cyclic guanosine monophosphate, $c G M P / C r$ cGMP normalized to creatinine, $I Q R$ interquartile range

The changes in AGV from baseline and CXM were dosedependent from 2.5 to $30 \mu \mathrm{g} / \mathrm{kg}$, and exposure-response analyses showed that both have reached the plateau of the exposure-response curve at exposures obtained at $15 \mu \mathrm{g}$ / 


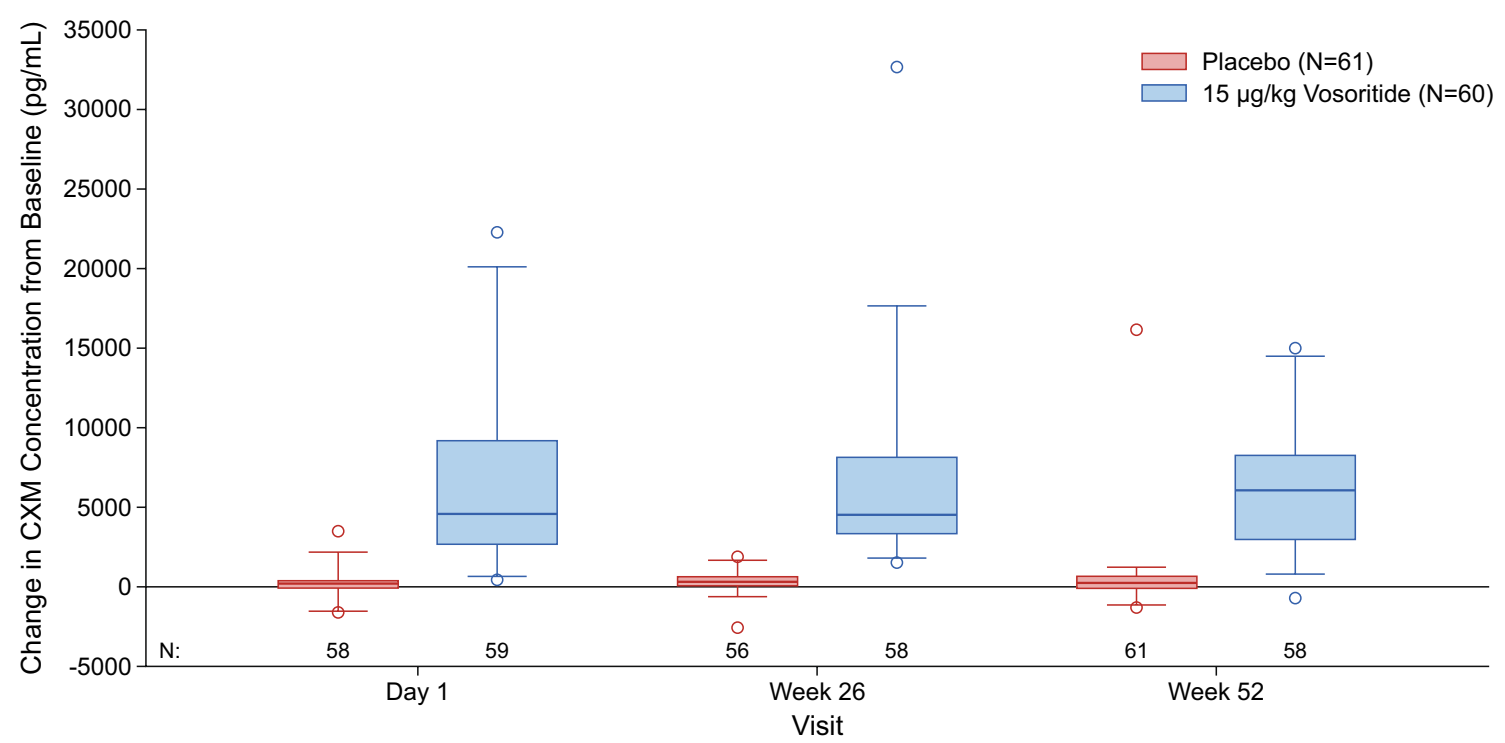

Fig. 6 Distribution of the change in CXM from baseline from week 13 to week 52 of the phase III study. The line inside the box represents the median and the box represents the limits of the middle half of the data. The range of the box, from the first quartile (Q1) to the third quartile $(\mathrm{Q} 3)$, defines the IQR. The standard span of the data is defined within the range from Q1 $-1.5 \times$ IQR to $\mathrm{Q} 3+1.5 \times \mathrm{IQR}$. Whiskers are drawn to the nearest value not beyond the range of the standard span; points beyond are drawn as individual open circles. $C X M$ collagen type $\mathrm{X}$ marker, $I Q R$ interquartile range

Table 5 Sigmoid $E_{\max }$ model parameter estimates for urine cGMP/Cr (initial 6 months of the phase II study)

\begin{tabular}{lllll}
\hline $\begin{array}{l}\text { Pharmacokinetic } \\
\text { parameter }\end{array}$ & $\begin{array}{l}E_{0}(\mathrm{pmol} / \mathrm{mg} \mathrm{Cr}) \\
\text { MPE }(95 \% \mathrm{CI})\end{array}$ & $\begin{array}{l}E_{\max }(\mathrm{pmol} / \mathrm{mg} \mathrm{Cr}) \\
\text { MPE }(95 \% \mathrm{CI})\end{array}$ & $\begin{array}{l}E C_{50} \\
\text { MPE (95\% CI) }\end{array}$ & $\begin{array}{l}h \\
\text { MPE (95\% CI) }\end{array}$ \\
\hline$C_{\max }$ & $579(-331$ to 1490$)$ & $10,100(7720-12,600)$ & $\begin{array}{l}4790 \mathrm{pg} / \mathrm{mL}(3270-6320) \\
2.04(0.811-3.26)\end{array}$ \\
$\mathrm{AUC}_{0-\mathrm{t}}$ & $393(-503$ to 1290$)$ & $11,800(8140-15,400)$ & $\begin{array}{l}2.22 \times 10^{5} \mathrm{pg}-\mathrm{min} / \mathrm{mL}\left(7.48 \times 10^{4}\right. \\
\left.\text { to } 3.69 \times 10^{5}\right)\end{array}$ & $1.12(0.486-1.76)$ \\
\hline
\end{tabular}

$C_{\max }$ maximum observed plasma concentration, $A U C_{0-t}$ area under the plasma concentration-time curve from time zero to the time of the last measurable concentration, $C I$ confidence interval, $E_{0}$ baseline level, $E C_{50}$ pharmacokinetic parameter value producing half maximal effect on cGMP/Cr, $E_{\max }$ maximum possible effect, $c G M P$ cyclic guanosine monophosphate, $c G M P / C r$ cGMP normalized to creatinine, $h$ Hill coefficient, $M P E$ model parameter estimates

Table $6 E_{\max }$ model parameter estimates for urine cGMP/Cr in the phase III study

\begin{tabular}{|c|c|c|c|}
\hline $\begin{array}{l}\text { Pharmacoki- } \\
\text { netic parameter }\end{array}$ & $\begin{array}{l}E_{0}(\mathrm{pmol} / \mathrm{mg} \mathrm{Cr}) \\
\operatorname{MPE}(95 \% \mathrm{CI})\end{array}$ & $\begin{array}{l}E_{\max }(\mathrm{pmol} / \mathrm{mg} \mathrm{Cr}) \\
\operatorname{MPE}(95 \% \mathrm{CI})\end{array}$ & $\begin{array}{l}E C_{50} \\
\operatorname{MPE}(95 \% \mathrm{CI})\end{array}$ \\
\hline$C_{\max }$ & -3014 (- 8800 to 2770$)$ & $18,160(13,800-22,600)$ & $5020 \mathrm{pg} / \mathrm{mL}(-714$ to 10,800$)$ \\
\hline $\mathrm{AUC}_{0-60 \mathrm{~min}}$ & $4.54(-2730$ to 2740$)$ & $21,900(11,800-32,000)$ & $\begin{array}{l}4.79 \times 10^{5} \mathrm{pg}-\mathrm{min} / \mathrm{mL}(-9.25 \\
\left.\quad \times 10^{4}, 1.05 \times 10^{6}\right)\end{array}$ \\
\hline
\end{tabular}

$C_{\max }$ maximum observed plasma concentration, $A U C_{0-60 \min }$ area under the plasma concentration-time curve from time zero to $60 \mathrm{~min}$ postdose, $C I$ confidence interval, $E_{0}$ baseline level, $E C_{50}$ pharmacokinetic parameter value producing half maximal effect on $\mathrm{cGMP} / \mathrm{Cr}, E_{\max }$ maximum possible effect, $c G M P$ cyclic guanosine monophosphate, $c G M P / C r$ cGMP normalized to creatinine, MPE model parameter estimates $\mathrm{kg}$. There was no additional benefit in effect on AGV or CXM seen with the $30 \mu \mathrm{g} / \mathrm{kg}$ daily dose compared with $15 \mu \mathrm{g} / \mathrm{kg}$. Exposure-cGMP response analysis showed that systemic pharmacological activity is near maximal or saturated at exposures obtained at $30 \mu \mathrm{g} / \mathrm{kg}$. Given that exposure-response relationship for changes in both AGV and CXM saturated at $15 \mu \mathrm{g} / \mathrm{kg}$, the additional systemic pharmacological activity indicated by the increase in urine 
a

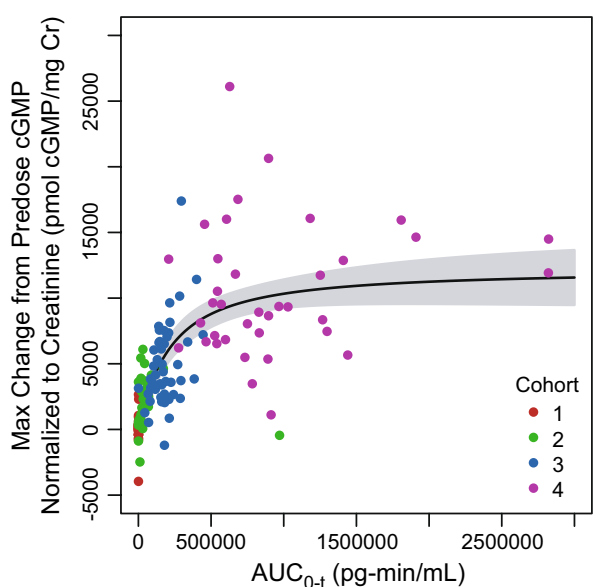

Fig. 7 Visit-matched maximum increase in urine cGMP/Cr by individual AUC in the phase II and III studies. a Points represent the individual patient urine cGMP/Cr data and individual plasma vosoritide $\mathrm{AUC}_{0-\mathrm{t}}$ at each visit during the initial 6 months of the phase II study, color-coded by study cohort (C1: Cohort $1,2.5 \mu \mathrm{g} / \mathrm{kg}$; C2: Cohort 2, $7.5 \mu \mathrm{g} / \mathrm{kg}$; C3: Cohort 3, $15 \mu \mathrm{g} / \mathrm{kg}$; C4: Cohort 4, $30 \mu \mathrm{g} / \mathrm{kg}$ ). One patient had a high $\mathrm{AUC}_{0-\mathrm{t}}\left(1.63 \times 10^{7} \mathrm{pg}-\mathrm{min} / \mathrm{mL}\right)$ relative to other patients, but a within-range maximum change from pre-dose cGMP/ $\mathrm{Cr}(8190 \mathrm{pmol} / \mathrm{mg} \mathrm{Cr})$. This patient was included in the fit through the data but was not included in this figure. b Points represent the individual patient urine $\mathrm{cGMP} / \mathrm{Cr}$ data and individual plasma voso- b

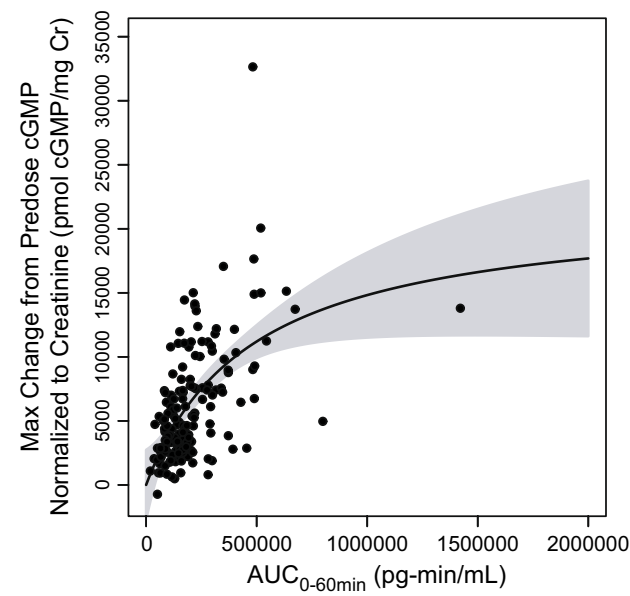

ritide $\mathrm{AUC}_{0-60 \mathrm{~min}}$ at each visit during the phase III study. Solid lines represent $E_{\max }$ model fits through the data and the shaded regions represent the $95 \%$ confidence intervals. The respective analyses using the individual plasma vosoritide $C_{\max }$ are similar and are shown in Fig. $\mathrm{S} 7$ in the ESM. $c G M P$ cyclic guanosine monophosphate, $c G M P / C r$ cGMP normalized to creatinine, $A U C_{0-t}$ area under the plasma concentration-time curve from time zero to the time of the last measurable concentration, $A U C_{0-60 \text { min }}$ area under the plasma concentration-time curve from time zero to $60 \mathrm{~min}$ postdose, $E_{\max }$ maximum possible effect, ESM electronic supplementary material a

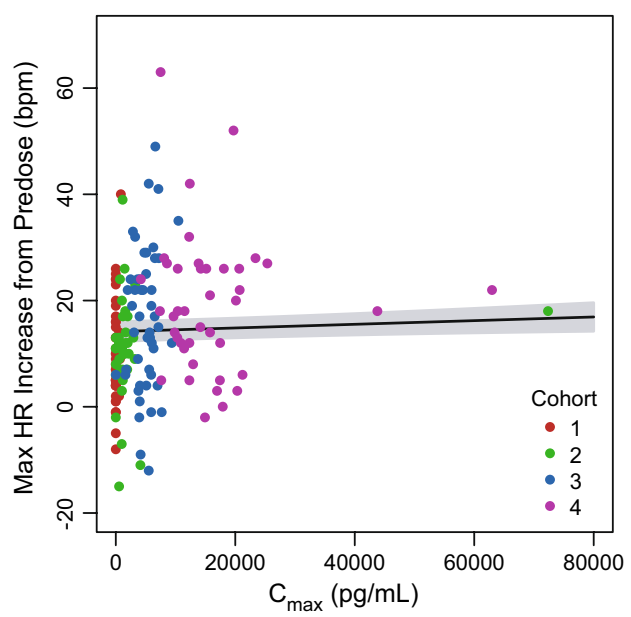

Fig. 8 Visit-matched vosoritide $C_{\max }$ in plasma and maximum increase in heart rate in the phase II and III studies. a Points represent the individual patient maximum increase in HR and individual plasma vosoritide $C_{\max }$ at each visit during the initial 6 months of the phase II study, color-coded by study cohort (C1: Cohort 1, $2.5 \mu \mathrm{g} /$ $\mathrm{kg}$; C2: Cohort 2, $7.5 \mu \mathrm{g} / \mathrm{kg}$; C3: Cohort 3, $15 \mu \mathrm{g} / \mathrm{kg}$; C4: Cohort 4, $30 \mu \mathrm{g} / \mathrm{kg})$. One patient had a high $C_{\max }\left(8.39 \times 10^{5} \mathrm{pg} / \mathrm{mL}\right)$ relative to other patients, but a within-range maximum increase in HR (39 bpm). This patient was included in the fit through the data but was b

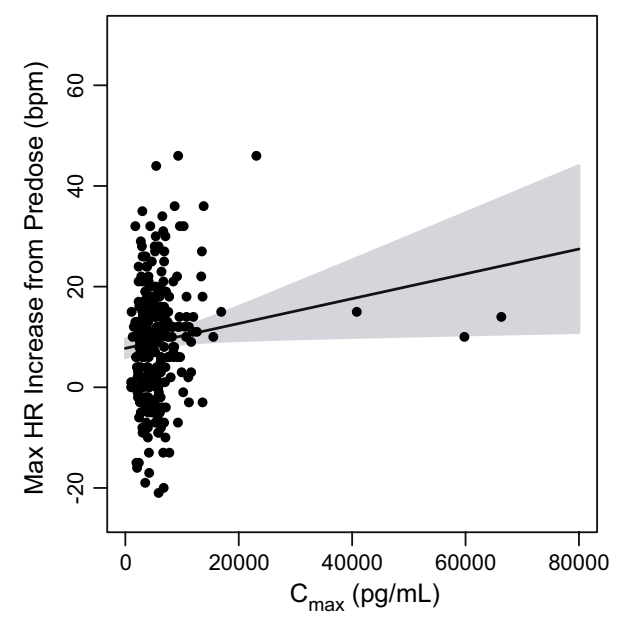

not included in this figure. b Points represent the individual patient maximum increase in HR and individual plasma vosoritide $C_{\max }$ at each visit during the phase III study. Solid lines represent the linear fits through the data and the shaded regions represent the $95 \%$ confidence interval. The respective analyses using the individual plasma vosoritide AUC is similar and is shown in Fig. S8 in the ESM. $C_{\max }$ maximum observed plasma concentration, $H R$ heart rate, $b p m$ beats per min, $A U C$ area under the plasma concentration-time curve, ESM electronic supplementary material 
$\mathbf{a}$

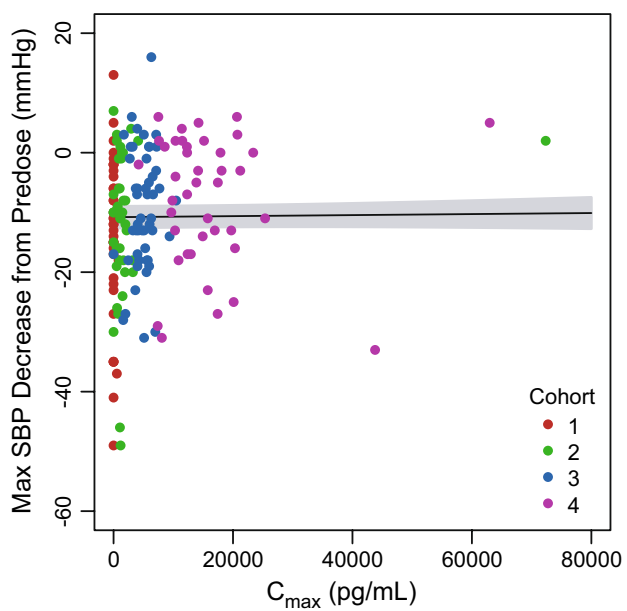

c

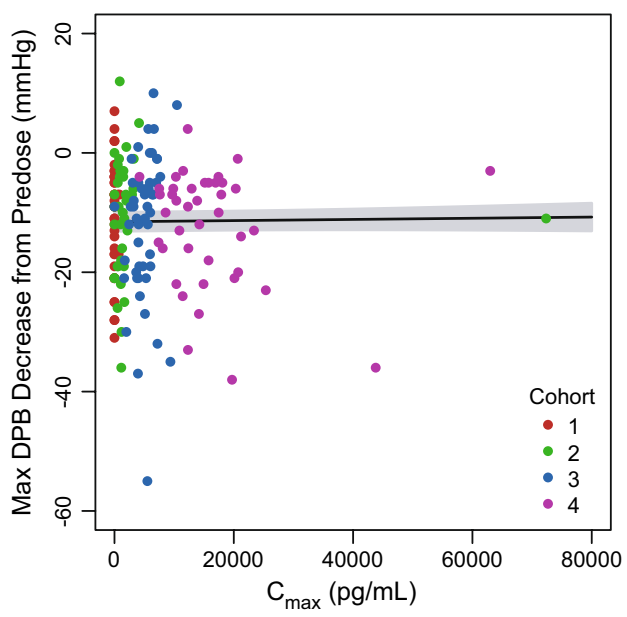

Fig. 9 Visit-matched vosoritide $C_{\max }$ in plasma and maximum decrease in systolic and diastolic blood pressures in the phase II and III studies. a Points represent the individual patient maximum decrease in SBP and individual plasma vosoritide $C_{\max }$ at each visit during the initial 6 months of the phase II study, color-coded by study cohort (C1: Cohort $1,2.5 \mu \mathrm{g} / \mathrm{kg}$; $\mathrm{C} 2$ : Cohort $2,7.5 \mu \mathrm{g} / \mathrm{kg}$; C3: Cohort 3, $15 \mu \mathrm{g} / \mathrm{kg}$; C4: Cohort 4, $30 \mu \mathrm{g} / \mathrm{kg}$ ). One patient had a high $C_{\max }\left(8.39 \times 10^{5} \mathrm{pg} / \mathrm{mL}\right)$ relative to other patients, but a within-range maximum decrease in SBP $(-7 \mathrm{mmHg})$. This patient was included in the fit through the data but was not included in this figure. b Points represent the individual patient maximum decrease in SBP and individual plasma vosoritide $C_{\max }$ at each visit during the phase III study. c Points represent the individual patient maximum decrease in DBP and individual plasma vosoritide $C_{\max }$ at each visit during the ini-

cGMP at the higher end of the exposure range (i.e., exposure from $30 \mu \mathrm{g} / \mathrm{kg}$ ) was likely in tissues not related to endochondral bone formation. Besides chondrocytes in the growth plates, NPR-B is expressed in many organs, including the heart, brain, liver, lungs and adipocytes [29, 39], while CXM is specifically produced by hypertrophic chondrocytes during endochondral bone formation. As such, cGMP levels are not specific to bone but reflect systemic activity in all tissues b

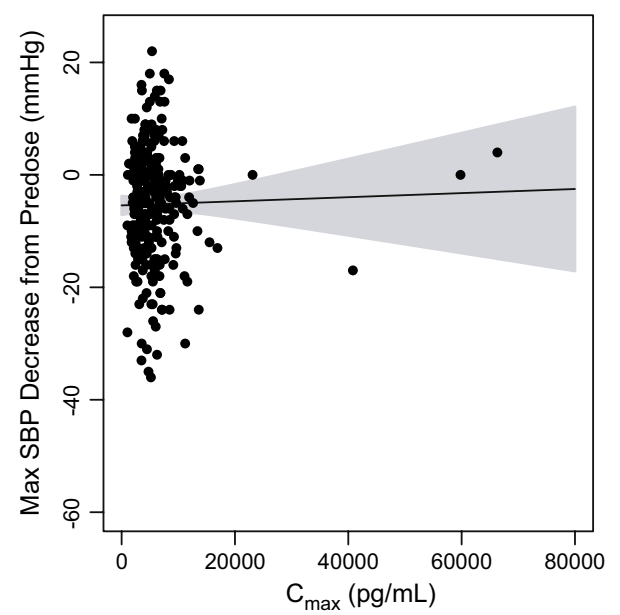

d

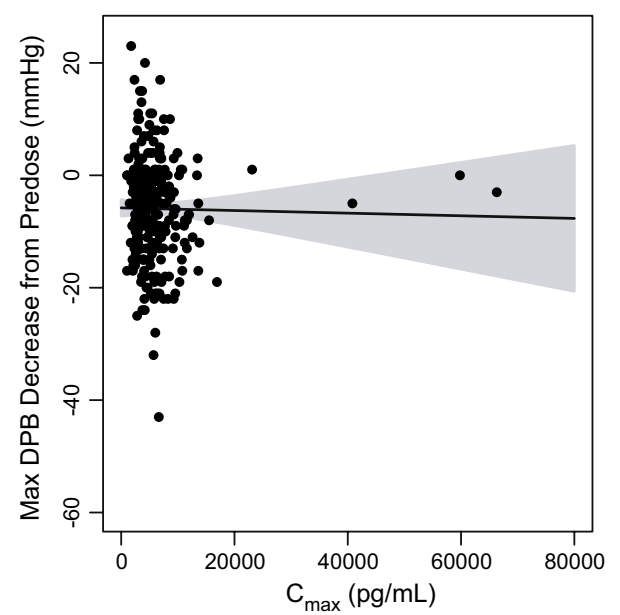

tial 6 months of the phase II study, color-coded by study cohort $(\mathrm{C} 1$ : Cohort 1, $2.5 \mu \mathrm{g} / \mathrm{kg}$; C2: Cohort 2, $7.5 \mu \mathrm{g} / \mathrm{kg}$; C3: Cohort 3, $15 \mu \mathrm{g} /$ $\mathrm{kg}$; C4: Cohort $4,30 \mu \mathrm{g} / \mathrm{kg})$. One patient had a high $C_{\max }(8.39 \times$ $10^{5} \mathrm{pg} / \mathrm{mL}$ ) relative to other patients, but a within-range maximum decrease in DBP $(-2 \mathrm{mmHg})$. This patient was included in the fit through the data but was not included in this figure. $\mathbf{d}$ Points represent the individual patient maximum decrease in DBP and individual plasma vosoritide $C_{\max }$ at each visit during the phase III study. Solid lines represent the linear fits through the data and the shaded regions represent the $95 \%$ confidence interval. The respective analyses using the individual patient AUC are similar and are shown in Fig. S9 in the ESM. $C_{\max }$ maximum observed plasma concentration, SBP systolic blood pressure, $\mathrm{DBP}$ diastolic blood pressure, $\mathrm{mmHg}$ millimeter of mercury, ESM electronic supplementary material

expressing NPR-B. Hence, CXM could become saturated at the growth plates at lower doses compared with systemic cGMP.

In addition to monitoring heart rate as well as systolic and diastolic blood pressures in the clinic, the analysis showed that there were no clinically meaningful correlations between vosoritide plasma exposure and these safety endpoints at clinically relevant exposures. This supports a 
wide therapeutic index for vosoritide within which vosoritide can be administered safely and efficaciously to children with achondroplasia.

\section{Conclusion}

The PK of vosoritide and exposure-response relationships with efficacy, biomarkers and safety endpoints were characterized in children with achondroplasia. Results support the recommended dose of $15 \mu \mathrm{g} / \mathrm{kg}$ vosoritide for once-daily subcutaneous administration in patients with achondroplasia aged $\geq 5$ years whose epiphyses were not closed.

Supplementary Information The online version contains supplementary material available at https://doi.org/10.1007/s40262-021-01059-1.

Acknowledgments The authors are grateful to the patients and their families for participating in these studies, and the study personnel at the various clinical sites and other co-workers for the provision of the data for the analyses, as well as Terry Manspeaker for assistance with the figures.

Author Contributions MLC performed the PK and exposure-response analyses and wrote the first draft of the manuscript. RS, MI, CAB, JH-F, KO, KM and WRW recruited and enrolled patients to the trials and managed them during the trial periods according to the protocols. WAH collaborated with BioMarin to develop its type X collagen assay. All authors participated in critical review of the manuscript and approved the final draft for submission.

Funding These studies were funded by BioMarin Pharmaceutical Inc.

\section{Declarations}

Conflict of Interest Ming Liang Chan, Yulan Qi, Kevin Larimore, Anu Cherukuri, Lori Seid, Kala Jayaram, Elena Fisheleva, Jonathan Day, Alice Huntsman Labed and Joshua Henshaw are employees of BioMarin Pharmaceutical Inc. Ravi Savarirayan and Klaus Mohnike have received consulting fees and grants from BioMarin. Melita Irving has received consulting fees from BioMarin. Carlos A. Bacino has received consulting fees, honoraria and grants from BioMarin. Julie Hoover-Fong has received consulting fees from BioMarin, Therachon and Ascendis, and grants from BioMarin. Keiichi Ozono has received consulting fees and honoraria from BioMarin. William R. Wilcox was a consultant to BioMarin and received an honoraria, and was the principal investigator for the clinical trial contract with Emory University. William A. Horton is an inventor on a patent application "Type $\mathrm{X}$ collagen assay and methods of use thereof", submitted by Shriners Hospitals for Children. He has consulted for and/or received speaker honoraria from BioMarin, TherAchon (Pfizer), Ascendis, QED, Relay Therapeutics, Fortress Biotech, OPKO, and Medicell Technologies.

Ethics Approval The studies were conducted in accordance with the provisions of the Declaration of Helsinki. The study protocols were approved by the relevant ethics boards at each site.

Consent to Participate Written informed consent from a parent or legal guardian of each patient was obtained, and assent was obtained from patients, if appropriate, prior to enrolment.
Availability of Data and Material The de-identified individual participant data that underlie the results reported in this article (including text, tables, figures, and appendices) will be made available together with the research protocol and data dictionaries, for non-commercial, academic purposes. Additional supporting documents may be available on request. Investigators will be able to request access to these data, along with supporting documents, via a website beginning at 6 months and ending 2 years after publication. Data associated with any ongoing development program will be made available within 6 months after approval of the relevant product. Requests must include a research proposal clarifying how the data will be used, including proposed analysis methodology. Research proposals will be evaluated relative to publicly available criteria at the BioMarin website to determine whether access will be given, contingent on execution of a data access agreement with BioMarin Pharmaceutical. For data access requests, see www.BioMa rin.com

Consent for Publication Not applicable.

Code Availability Not applicable.

Open Access This article is licensed under a Creative Commons Attribution-NonCommercial 4.0 International License, which permits any non-commercial use, sharing, adaptation, distribution and reproduction in any medium or format, as long as you give appropriate credit to the original author(s) and the source, provide a link to the Creative Commons licence, and indicate if changes were made. The images or other third party material in this article are included in the article's Creative Commons licence, unless indicated otherwise in a credit line to the material. If material is not included in the article's Creative Commons licence and your intended use is not permitted by statutory regulation or exceeds the permitted use, you will need to obtain permission directly from the copyright holder. To view a copy of this licence, visit http://creativecommons.org/licenses/by-nc/4.0/.

\section{References}

1. Merker A, Neumeyer L, Hertel NT, Grigelioniene G, Mohnike K, Hagenäs L. Development of body proportions in achondroplasia: Sitting height, leg length, arm span, and foot length. Am J Med Genet A. 2018;176(9):1819-29.

2. Vajo Z, Francomano CA, Wilkin DJ. The molecular and genetic basis of fibroblast growth factor receptor 3 disorders: the achondroplasia family of skeletal dysplasias, muenke craniosynostosis, and crouzon syndrome with acanthosis nigricans. Endocr Rev. 2000;21(1):23-39.

3. Wright MJ, Irving MD. Clinical management of achondroplasia. Arch Dis Child. 2012;97(2):129-34.

4. Foldynova-Trantirkova S, Wilcox WR, Krejci P. Sixteen years and counting: The current understanding of fibroblast growth factor receptor 3 (fgfr3) signaling in skeletal dysplasias. Hum Mutat. 2011;33(1):29-41.

5. Horton WA, Hall JG, Hecht JT. Achondroplasia Lancet. 2007;370(9582):162-72.

6. Krakow D. Skeletal dysplasias. Clin Perinatol. 2015;42(2):301-19.

7. Alade Y, Tunkel D, Schulze K, McGready J, Jallo G, Ain M, et al. Cross-sectional assessment of pain and physical function in skeletal dysplasia patients. Clin Genet. 2013;84(3):237-43.

8. Hunter AG, Bankier A, Rogers JG, Sillence D, Scott CI. Medical complications of achondroplasia: a multicentre patient review. J Med Genet. 1998;35(9):705-12.

9. Ireland PJ, Johnson S, Donaghey S, Johnston L, McGill J, Zankl A, et al. Developmental milestones in infants and young 
australasian children with achondroplasia. J Dev Behav Pediatr. 2010;31(1):41-7.

10. Mahomed NN, Spellmann M, Goldberg MJ. Functional health status of adults with achondroplasia. Am J Med Genet. 1998;78(1):30-5.

11. Ranke MB, Wit JM. Growth hormone-past, present and future. Nat Rev Endocrinol. 2018;14(5):285-300.

12. Harada D, Namba N, Hanioka Y, Ueyama K, Sakamoto N, Nakano $\mathrm{Y}$, et al. Final adult height in long-term growth hormone-treated achondroplasia patients. Eur J Pediatr. 2017;176(7):873-9.

13. Kanaka-Gantenbein C. Present status of the use of growth hormone in short children with bone diseases (diseases of the skeleton). J Pediatr Endocrinol Metab. 2001;14(1):17-26.

14. Miccoli M, Bertelloni S, Massart F. Height outcome of recombinant human growth hormone treatment in achondroplasia children: a meta-analysis. Horm Res Paediatr. 2016;86(1):27-34.

15. Donaldson J, Aftab S, Bradish C. Achondroplasia and limb lengthening: results in a UK cohort and review of the literature. J Orthop. 2015;12(1):31-4.

16. Yasoda A, Kazuwa N. Translational research of c-type natriuretic peptide (CNP) into skeletal dysplasias. Endocr J. 2010;57(8):659-66.

17. Kozhemyakina E, Lassar AB, Zelzer E. A pathway to bone: Signaling molecules and transcription factors involved in chondrocyte development and maturation. Development. 2015;142(5):817-31.

18. Chusho H, Tamura N, Ogawa Y, Yasoda A, Suda M, Miyazawa T, et al. Dwarfism and early death in mice lacking c-type natriuretic peptide. Proc Natl Acad Sci USA. 2001;98(7):4016-21.

19. Tamura N, Doolittle LK, Hammer RE, Shelton JM, Richardson JA, Garbers DL. Critical roles of the guanylyl cyclase b receptor in endochondral ossification and development of female reproductive organs. Proc Natl Acad Sci USA. 2004;101(49):17300-5.

20. Bocciardi R, Giorda R, Buttgereit J, Gimelli S, Divizia MT, Beri $S$, et al. Overexpression of the c-type natriuretic peptide (CNP) is associated with overgrowth and bone anomalies in an individual with balanced $\mathrm{t}(2 ; 7)$ translocation. Hum Mutat. 2007;28(7):724-31.

21. Kake T, Kitamura H, Adachi Y, Yoshioka T, Watanabe T, Matsushita $\mathrm{H}$, et al. Chronically elevated plasma c-type natriuretic peptide level stimulates skeletal growth in transgenic mice. Am J Physiol Endocrinol Metabol. 2009;297(6):E1339-48.

22. Ko JM, Bae J-S, Choi JS, Miura K, Lee HR, Kim O-H, et al. Skeletal overgrowth syndrome caused by overexpression of c-type natriuretic peptide in a girl with balanced chromosomal translocation, $\mathrm{t}(1 ; 2)(\mathrm{q} 41 ; \mathrm{q} 37.1)$. Am J Med Genet A. 2015;167A(5):1033-8.

23. Moncla A, Missirian C, Cacciagli P, Balzamo E, Legeai-Mallet L, Jouve J-L, et al. A cluster of translocation breakpoints in $2 q 37$ is associated with overexpression of NPPC in patients with a similar overgrowth phenotype. Hum Mutat. 2007;28(12):1183-8.

24. Wendt DJ, Dvorak-Ewell M, Bullens S, Lorget F, Bell SM, Peng $\mathrm{J}$, et al. Neutral endopeptidase-resistant c-type natriuretic peptide variant represents a new therapeutic approach for treatment of fibroblast growth factor receptor 3-related dwarfism. J Pharmacol Exp Ther. 2015;353(1):132-49.

25. Yasoda A, Kitamura H, Fujii T, Kondo E, Murao N, Miura M, et al. Systemic administration of c-type natriuretic peptide as a novel therapeutic strategy for skeletal dysplasias. Endocrinology. 2009;150(7):3138-44.

26. Yasoda A, Komatsu Y, Chusho H, Miyazawa T, Ozasa A, Miura M, et al. Overexpression of cnp in chondrocytes rescues achondroplasia through a mapk-dependent pathway. Nat Med. 2004;10(1):80-6.
27. Krejci P, Masri B, Fontaine V, Mekikian PB, Weis M, Prats H, et al. Interaction of fibroblast growth factor and c-natriuretic peptide signaling in regulation of chondrocyte proliferation and extracellular matrix homeostasis. J Cell Sci. 2005;118(21):5089-100.

28. Lorget F, Kaci N, Peng J, Benoist-Lasselin C, Mugniery E, Oppeneer T, et al. Evaluation of the therapeutic potential of a cnp analog in a fgfr 3 mouse model recapitulating achondroplasia. Am J Hum Genet. 2012;91(6):1108-14.

29. Potter LR, Yoder AR, Flora DR, Antos LK, Dickey DM. Natriuretic peptides: Their structures, receptors, physiologic functions and therapeutic applications. Handb Exp Pharmacol. 2009;191:341-66.

30. Coghlan RF, Oberdorf JA, Sienko S, Aiona MD, Boston BA, Connelly KJ, et al. A degradation fragment of type $\mathrm{x}$ collagen is a real-time marker for bone growth velocity. Sci Transl Med. 2017;9(419):eaan4669.

31. Shen $\mathrm{G}$. The role of type $\mathrm{x}$ collagen in facilitating and regulating endochondral ossification of articular cartilage. Orthod Craniofac Res. 2005;8(1):11-7.

32. Coghlan RF, Olney RC, Boston BA, Coleman DT, Johnstone B, Horton WA. Norms for clinical use of CXM, a real-time marker of height velocity. J Clin Endocrinol Metab. 2021;106(1):e255-64.

33. Savarirayan R, Irving M, Bacino CA, Bostwick B, Charrow J, Cormier-Daire V, et al. C-type natriuretic peptide analogue therapy in children with achondroplasia. New Engl J Med. 2019;381(1):25-35.

34. Savarirayan RTL, Irving M, Wilcox W, Bacino CA, HooverFong J, Font RU, et al. Once-daily, subcutaneous vosoritide therapy in children with achondroplasia: a randomised, doubleblind, phase 3, placebo-controlled, multicentre trial. Lancet. 2020;396(10252):684-92.

35. Li D. Strategic approaches to optimizing peptide adme properties. AAPS J. 2015;17(1):134-43.

36. Diao L, Meibohm B. Pharmacokinetics and pharmacokineticpharmacodynamic correlations of therapeutic peptides. Clin Pharmacokinet. 2013;52(10):855-68.

37. Potter LR. Natriuretic peptide metabolism, clearance and degradation. FEBS J. 2011;278(11):1808-17.

38. Meibohm B. Pharmacokinetics and pharmacodynamics of peptide and protein therapeutics. In: Crommelin DJA, Sindelar RD, Meibohm B, editors. Pharmaceutical biotechnology. New York: Springer; 2013. p. 101-32.

39. Santhekadur PK, Kumar DP, Seneshaw M, Mirshahi F, Sanyal AJ. The multifaceted role of natriuretic peptides in metabolic syndrome. Biomed Pharmacother. 2017;92:826-35.

40. Chirmule N, Jawa V, Meibohm B. Immunogenicity to therapeutic proteins: Impact on $\mathrm{pk} / \mathrm{pd}$ and efficacy. AAPS J. 2012;14(2):296-302.

41. Haymond M, Kappelgaard A-M, Czernichow P, Biller BM, Takano K, Kiess W, et al. Early recognition of growth abnormalities permitting early intervention. Acta Paediatr. 2013;102(8):787-96.

42. del Pino M, Fano V, Adamo P. Growth in achondroplasia, from birth to adulthood, analysed by the jpa-2 model. J Pediatr Endocrinol Metab. 2020;33(12):1589-95.

43. Hoover-Fong JE, Schulze KJ, McGready J, Barnes H, Scott CI. Age-appropriate body mass index in children with achondroplasia: interpretation in relation to indexes of height. Am J Clin Nutr. 2008;88(2):364-71.

44. Madsen A, Fredwall SO, Maanum G, Henriksen C, Slettahjell HB. Anthropometrics, diet, and resting energy expenditure in norwegian adults with achondroplasia. Am J Med Genet A. 2019;179(9):1745-55. 


\section{Authors and Affiliations}

Ming Liang Chan ${ }^{1}$ D $\cdot$ Yulan $\mathrm{Qi}^{1} \cdot$ Kevin Larimore $^{1} \cdot$ Anu Cherukuri $^{1} \cdot$ Lori Seid $^{1} \cdot$ Kala Jayaram $^{1} \cdot$ George Jeha $^{1}$. Elena Fisheleva ${ }^{2}$ Jonathan Day ${ }^{2}$ Alice Huntsman-Labed ${ }^{2} \cdot$ Ravi Savarirayan $^{3} \cdot$ Melita Irving $^{4} \cdot$ Carlos A. Bacino $^{5}$. Julie Hoover-Fong ${ }^{6} \cdot$ Keiichi Ozono $^{7} \cdot$ Klaus Mohnike $^{8} \cdot$ William R. Wilcox $^{9} \cdot$ William A. Horton $^{10} \cdot$ Joshua Henshaw $^{1}$

1 BioMarin Pharmaceutical Inc, Novato, CA, USA

2 BioMarin (UK) Limited, London, UK

3 Murdoch Children's Research Institute, Royal Children's Hospital, University of Melbourne, Parkville, VIC, Australia

4 Guy's and St. Thomas' NHS Foundation Trust, Evelina Children's Hospital, London, UK

5 Baylor College of Medicine, Houston, TX, USA
6 Greenberg Center for Skeletal Dysplasias, Department of Genetic Medicine, Johns Hopkins University School of Medicine, Baltimore, MD, USA

7 Osaka University Hospital, Osaka, Japan

8 Otto-von-Guericke-Universität, Magdeburg, Germany

9 Department of Human Genetics, Emory University, Atlanta, GA, USA

10 Shriners Hospitals for Children, Portland, OR, USA 